\title{
Hybrid Multiscale Simulation of a Mixing-Controlled Reaction
}

\author{
Timothy D. Scheibe, ${ }^{a^{*}}$ Karen Schuchardt, ${ }^{a}$ Khushbu Agarwal, ${ }^{a}$ Jared Chase, ${ }^{a}$ Xiaofan \\ Yang, ${ }^{\text {a } B r u c e ~ J . ~ P a l m e r, ~}{ }^{\mathrm{a}}$ Alexandre M. Tartakovsky, ${ }^{\mathrm{a}}$ Todd Elsethagen, ${ }^{\mathrm{a}}$ George \\ Redden $^{\mathrm{b}}$ \\ ${ }^{a}$ Pacific Northwest National Laboratory, PO Box 999, MS K9-36, Richland, WA 99352 \\ ${ }^{b}$ Montana State University, Bozeman, MT 59717 \\ * Corresponding author. tel.: +1-509-372-6065 ; fax: +1-509-372-6089 . \\ E-mail address: Tim.Scheibe@pnnl.gov
}

Elsevier use only: Received date here; revised date here; accepted date here

\begin{abstract}
Continuum-scale models, which employ a porous medium conceptualization to represent properties and processes averaged over a large number of solid grains and pore spaces, are widely used to study subsurface flow and reactive transport. Recently, pore-scale models, which explicitly resolve individual soil grains and pores, have been developed to more accurately model and study pore-scale phenomena, such as mineral precipitation and dissolution reactions, microbially-mediated surface reactions, and other complex processes. However, these highly-resolved models are prohibitively expensive for modeling domains of sizes relevant to practical problems. To broaden the utility of pore-scale models for larger domains, we developed a hybrid multiscale model that initially simulates the full domain at the continuum scale and applies a pore-scale model only to areas of high reactivity. Since the location and number of pore-scale model regions in the model varies as the reactions proceed, an adaptive script defines the number and location of pore regions within each continuum iteration and initializes pore-scale simulations from macroscale information. Another script communicates information from the pore-scale simulation results back to the continuum scale. These components provide loose coupling between the pore- and continuum-scale codes into a single hybrid multiscale model implemented within the SWIFT workflow environment. In this paper, we consider an irreversible homogenous bimolecular reaction (two solutes reacting to form a third solute) in a $2 \mathrm{D}$ test problem. This paper is focused on the approach used for multiscale coupling between pore- and continuum-scale models, application to a realistic test problem, and implications of the results for predictive simulation of mixing-controlled reactions in porous media. Our results and analysis demonstrate that the hybrid multiscale method provides a feasible approach for increasing the accuracy of subsurface reactive transport simulations. (ㅇ 2014 Elsevier Science. All rights reserved.
\end{abstract}




\section{Introduction}

Granular porous media are made up of solid grains of variable size and shape with intervening pore spaces filled with one or more fluids (e.g., water, air, non-aqueous-phase liquids). However, because of the difficulty of characterizing properties and simulating processes at a scale that explicitly resolves these phases, conventional simulations of flow and reactive transport in porous media treat the medium as an effective continuum. The rates and directions of flow, transport and reaction are defined over a "representative elementary volume" (REV) of porous media for which the effects of variations in fluid flow and solute diffusion within individual pores are approximated by apparent or upscaled model forms and parameters (e.g., Darcy's law and the advection-dispersionreaction equation, parameterized by the hydraulic conductivity tensor, porosity, longitudinal and transverse dispersivities, and effective reaction rate models). Here, we refer to models that rely on this conceptualization as continuum-scale models. In contrast, it is also possible to explicitly simulate processes at the pore scale, at which the details of solid and pore geometry can be directly incorporated and velocity variations can be resolved at the scale of tens of micrometers or smaller (i.e., "pore-scale models). Well-developed pore-scale modeling methodologies include the Lattice-Boltzmann (LB) method [1-8], the Smoothed Particle Hydrodynamics (SPH) method [9-16], computational fluid dynamics (CFD) methods [17-26], and pore network models [27-38]. These models have proven useful for gaining new insights into the relationship between fundamental processes defined at the pore scale and upscaled phenomena observed at Darcy scales. Significant advances have been made in threedimensional (3D) characterization of porous media structure, using methods such as x-ray computed tomography (XCT) and magnetic resonance imaging (MRI), and quantitative descriptions of pore geometry with sufficient resolution to facilitate pore-scale simulations [39]. Simultaneous advances in high-performance computing have provided the computational power necessary to perform pore-scale simulations over complex 3D domains. However, despite these advances pore-scale simulations remain extremely computationally expensive, and have typically been performed on millimeter-scale or smaller domains, with the most advanced high-performance computing applications to date enabling simulations on centimeter- to decimeter-scale domains [17, 26, 40, 41]. Therefore, it is currently infeasible to perform simulations on domain sizes of practical interest (scales of meters to kilometers) with complete pore-scale resolution, and will likely remain so for some time to come.

Nevertheless, recent studies have pointed to the potential need for pore-scale resolution of processes for accurate simulation of some types of reactive transport problems [42, 43]. Of particular note are reactive transport processes in which reaction rates are not limited by fundamental molecular interactions, but instead are controlled by mixing processes (primarily diffusion) at interfaces between fluid and/or solid phases containing the various reactants [4446]. These mixing-controlled reactions are often characterized by sharp local gradients and localized reaction zones; example problems include biologically-mediated natural attenuation of dissolved contaminant plumes [47, 48], precipitation/dissolution reactions [10, 49,50], incomplete mixing effects on reaction rates [51-57]. and biofilm dynamics $[11,58]$. There have been many representative pore-scale modeling works on transverse mixingcontrolled reactive transport [58-66]. A brief review of mixing, spreading and reaction in heterogenous media has been given in [46]. Battiato and others [67-69] developed a multiscale analysis of a mixing-controlled precipitation reaction that identified sufficient conditions (in terms of ranges of dimensionless Péclet $(\mathrm{Pe})$ and Damköhler (Da) numbers) under which macroscopic advection-dispersion-reaction equations provided an accurate description of the pore-scale processes. Outside of these ranges, it was postulated that pore-scale processes must be explicitly simulated to obtain an accurate solution.

Problems in which explicit pore-scale simulation is essential, in the context of computational limitations of porescale simulation over large domains, motivate a hybrid multiscale simulation approach in which pore- and continuum-scale models are directly coupled within a single simulation domain. Scheibe et al. [70] provide a review of hybrid multiscale simulation methods and their applications in a number of scientific domains including recent developments in modeling of reactive transport in porous and fractured media. Tartakovsky and Scheibe [71] demonstrated an approach for coupling continuum- and pore-scale models applied to a mixing-controlled precipitation reaction over a small (mm-scale) domain. In this paper, we extend their approach to a much larger domain similar to the benchtop laboratory experiment described in [10]. We couple a pore-scale model based on the Smoothed Particle Hydrodynamics (SPH) code described in [72] with a continuum model based on the Subsurface Transport Over Multiple Phases (STOMP) simulator [73]. The study of [71] considered a single pore-scale domain corresponding to one continuum-scale grid element as a test case; here we extend this approach to consider multiple pore-scale SPH domains coupled to a two-dimensional array of STOMP grid elements representing a realistic problem of interest. While Tartakovsky and Scheibe [71] simulated a mixing-controlled bimolecular reversible 
mineral precipitation reaction, here for the sake of computational simplification we consider an irreversible homogenous bimolecular reaction (two solutes reacting to form a third solute) similar to the problem studied by [9]. We have developed a parallel workflow structure based on the Swift workflow environment $[74,75]$ that manages the complex process of executing many coupled pore- and continuum-scale code runs over the course of a single integrated multiscale hybrid simulation. Scheibe et al. [76] provide a description of the computational workflow management aspects of this work, which take advantage of multiple levels of parallelism inherent in the problem to scale the problem to a massively parallel supercomputing environment. Here, we focus on the approach used for multiscale coupling between pore- and continuum-scale models, application to a realistic test problem, and implications of the results for predictive simulation of mixing-controlled reactions in porous media.

\section{Methods}

In this section, we present the numerical methods for the pore-scale, continuum-scale and the hybrid multiscale models respectively, which include the governing equations, numerical methods, target problem and setup. The concept and the execution of the coupled hybrid workflow are introduced subsequently.

\subsection{Pore-scale simulation}

The Smoothed Particle Hydrodynamics (SPH) method is a fully Lagrangian mesh-free particle-based method, and directly solves discretized forms of the partial differential equations describing fluid flow and other porous media dynamics. It was initially developed for applications in astrophysics, but has recently been applied to a number of porous media flow and reactive transport problems [9-15]. SPH is also widely used for computergenerated animation used in gaming and commercial media, particularly to simulate fluid flows with free surfaces (e.g., [81, 82]). An overview of the method and applications is provided by [16]. Because discretization points ("particles") can move freely within the simulation domain, the SPH method is particularly well suited to simulation of problems that involve moving interfaces and dynamic pore geometry, including pore-scale precipitation/dissolution reactions. Although in this initial study we consider a solute-phase reaction only, which could be easily solved with a mesh-based method, we intend in future work to apply the same approach to a mixingcontrolled precipitation reaction system with dynamic pore geometry. Therefore, and because we have available inhouse a scalable parallel implementation of SPH [77], we chose the SPH method for our pore-scale simulator. However, we note that the methodology used here to couple pore- and continuum-scale simulations could be applied to other simulation methods and codes.

For purposes of testing the hybrid multiscale coupling approach, we chose to simulate a two-dimensional model system, both at pore- and continuum-scales. At the pore scale, the fundamental element is taken to be a $0.5 \mathrm{~cm}$ by $0.5 \mathrm{~cm}$ domain containing a regular pattern of uniform spherical grains as shown in Figure 1. This domain has periodic geometry, which facilitates handling of boundary conditions in the SPH simulations (described in greater detail below). The porosity of this domain is $\theta=0.446$, and the diameter of the spherical grains is $d_{g}=0.7 \mathrm{~mm}$. The system is discretized using $200 \times 200=40,000 \mathrm{SPH}$ particles, at an initial uniform spacing of $0.025 \mathrm{~mm}$, which provides a minimum resolution of five particles spanning the narrowest pore throats. A series of preliminary simulations were performed using the SPH code to define an appropriate parameterization. In particular, the applied body force ("gravity" parameter) was adjusted iteratively to obtain an average vertical velocity consistent with the macroscopic simulation, and other parameters (such as the stiffness parameter c) were selected to maximize computational stability and efficiency while maintaining water-like fluid properties. In these simulations, the Reynolds number of the system (Re) is less than unity, indicating creeping flow. Boundary conditions for flow are periodic; that is, any particle that leaves the domain is re-introduced at the corresponding point on the opposite boundary. Therefore, while the average horizontal velocity is zero, local fluxes can occur through the lateral boundaries as dictated by the local pore geometry. 


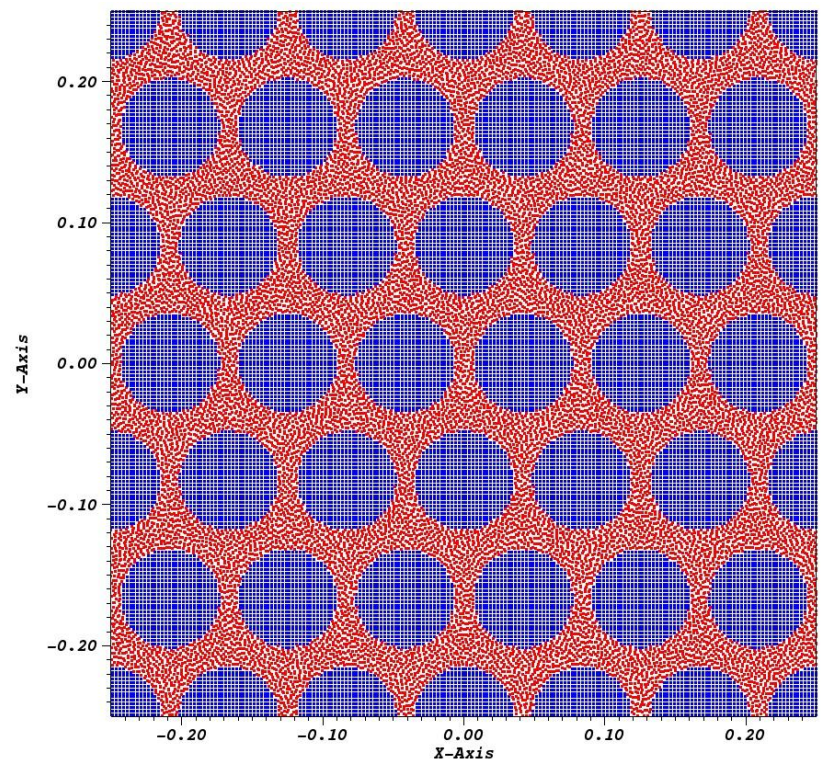

Figure 1 Idealized pore-scale geometry used for SPH simulations. Blue represents solid grains and red represents pore space.

We simulate a mixing-controlled reaction of two non-specific solutes (A and B) that react irreversibly to form a third solute $(\mathrm{C})$ according to the simple irreversible bimolecular reaction

$$
A+B \rightarrow C
$$

The reaction is assumed to be kinetically controlled, with the rate of the reaction given by

$$
d[C] / d t=k_{f}[A][B]
$$

where [*] denotes concentration of the dissolved species and $k_{f}$ is a constant reaction rate coefficient. Initial conditions are zero concentration of all species over the model domain; solutes A and B are introduced at the inflow boundary (bottom of the domain) by assigning concentrations of A and B to any particles introduced at the inlet (bottom) boundary according to a user-specified distribution provided in an input file. The input distribution is discrete in form, with a user-specified number of bins along the inlet boundary and uniquely-specified concentrations of $\mathrm{A}, \mathrm{B}$ and $\mathrm{C}$ as a function of location for each bin. For the SPH domain corresponding to the center grid element at the bottom boundary of the continuum simulation (described below), the concentrations are $\left(\mathrm{A}=A_{0}\right.$; $\mathrm{B}=0 ; \mathrm{C}=0)$ over the left half of the inlet boundary and $\left(\mathrm{A}=0 ; \mathrm{B}=B_{0} ; \mathrm{C}=0\right)$ over the right half, where $A_{0}$ and $B_{0}$ are constant input concentrations. For other SPH domains (corresponding to internal grid elements of the continuum simulation), the distribution of inlet concentrations is derived from concentration distributions in the grid element immediately below the element being simulated as described further below.

Impacts of mixing on this reaction system were previously studied at the pore scale by [9], who observed significant deviations from macroscopic predictions of reaction rates under certain conditions (ranges of nondimensional Reynolds (Re), Péclet (Pe) and Damköhler (Da) numbers as defined in [9]), suggesting the use of a hybrid pore/continuum modeling approach to simulate larger-scale problems such as the one under consideration here. The Péclet number is much greater than unity, indicating advection-dominated transport that emphasizes mixing limitations on reactions [9]. The Damköhler number reflects a diffusion-limited reaction system, again emphasizing the mixing-controlled nature of the reaction system. A summary of model parameters is provided in Table 1. 


\begin{tabular}{l|ccc}
\hline \multicolumn{1}{c|}{ Parameter } & Symbol(units) & Value & Unit \\
\hline SPH weighting function radius & $h$ & 0.01 & $\mathrm{~cm}$ \\
Grain diameter & $d_{g}$ & 0.07 & $\mathrm{~cm}$ \\
Darcy flux & $q$ & 0.01667 & $\mathrm{~cm} / \mathrm{s}$ \\
Average pore velocity & $v$ & 0.03737 & $\mathrm{~cm} / \mathrm{s}$ \\
Fluid density & $\rho$ & 1.0 & $\mathrm{~g} / \mathrm{cm}^{3}$ \\
Fluid viscosity & $\mu$ & 0.01 & $\mathrm{~g} / \mathrm{cm}-\mathrm{s}$ \\
SPH particle mass & $m_{0}$ & $6.25 \times 10^{-6}$ & $\mathrm{~g}$ \\
SPH domain dimension (length and width) & $L$ & 0.5 & $\mathrm{~cm}$ \\
Porosity & $\theta$ & 0.446 & -- \\
Molecular diffusion coefficient (in bulk water) & $d_{m}$ & $7.5 \times 10^{-6}$ & $\mathrm{~cm}^{2} / \mathrm{s}$ \\
Input concentration of reactants (A and B) & $A_{0}, B_{0}$ & $5.0 \times 10^{-5}$ & $\mathrm{moles} / \mathrm{cm}^{3}$ \\
Reaction rate coefficient & $k_{f}$ & 0.35 & \\
Applied body force & $g$ & 0.01962 & $\mathrm{~cm} / \mathrm{s}$ \\
Stiffness parameter (speed of sound) & $c$ & 16.0 & $\mathrm{~cm} / \mathrm{s}$ \\
Longitudinal dispersivity & $\alpha_{\mathrm{L}}$ & 0.05 & $\mathrm{~cm}$ \\
Transverse dispersivity & $\alpha_{\mathrm{T}}$ & 0.005 & $\mathrm{~cm}$ \\
Reynolds number & $\mathrm{Re}$ & 0.116 & -- \\
Péclet number & Pe & 155.49 & -- \\
Damköhler number & Da & 114.29 & -- \\
\hline
\end{tabular}

\subsection{Continuum-scale simulation}

The continuum-scale simulation is performed using the Subsurface Transport Over Multiple Phases (STOMP) simulator [73]. STOMP comprises many different operational modes depending on the problem being solved and has both serial and parallel (eSTOMP) versions; here we are considering only saturated flow and solute transport with aqueous-phase reactions in $2 \mathrm{D}$ on a modest number of grid nodes, and therefore we use the serial water-only mode with reactions (ECKEChem module). The model domain is $30.5 \mathrm{~cm}$ wide and $30 \mathrm{~cm}$ high, and is resolved on a uniform $0.5 \times 0.5 \mathrm{~cm}$ grid (Figure 2). Each grid element of STOMP therefore maps directly to one potential SPH pore-scale domain. The model system is patterned after the mixing-controlled reaction experiment reported in [10], but the specific conditions and reaction model are modified slightly for computational convenience. A specified flux boundary condition is imposed at the bottom of the domain, with a Darcy velocity of $1 \mathrm{~cm} / \mathrm{min}$, and a Dirichlet condition (specified pressure) is imposed at the top of the domain simulating the free outflow boundary of the experiment. Right and left boundaries are specified no-flow conditions. The porous material is assumed to be macroscopically homogeneous (uniform and isotropic hydraulic conductivity $\mathrm{K}_{\mathrm{x}}=\mathrm{K}_{\mathrm{y}}$ ) leading to uniform and steady macroscopic flow in the vertical (y) direction. Because the inlet boundary condition is specified flux, the macroscopic velocity does not depend on hydraulic conductivity, thus the value used does not affect the transport or reaction process. Tracer transport is assumed to follow the well-known advection-dispersion equation (ADE) with spatially uniform longitudinal and transverse dispersivities. The same reaction model as described above for the pore-scale simulation is used at the continuum scale, but the concentrations are defined as averages over each STOMP grid cell and therefore in some cases (as a result of imperfect mixing) the correct effective reaction rate coefficient differs from the constant (fully mixed) reaction rate coefficient specified at the pore scale. The method for specifying a spatially and temporally variable reaction rate coefficient in the STOMP model from pore scale simulations is described below in the model coupling section. In STOMP, this reaction system is implemented as the "forward-backward" reaction model, where the backward reaction rate is set to zero to simulate an irreversible reaction. For this study, the STOMP code was modified to allow prescription of spatially variable reaction rate coefficients $\left(k_{f}(\mathrm{i}, \mathrm{j})\right)$ through an user-specified input file (where $i$ and $j$ denote STOMP grid node indices in the $\mathrm{x}$ - and $\mathrm{y}$-axis directions, respectively), which is necessary for the coupling to the pore-scale model as described below. 


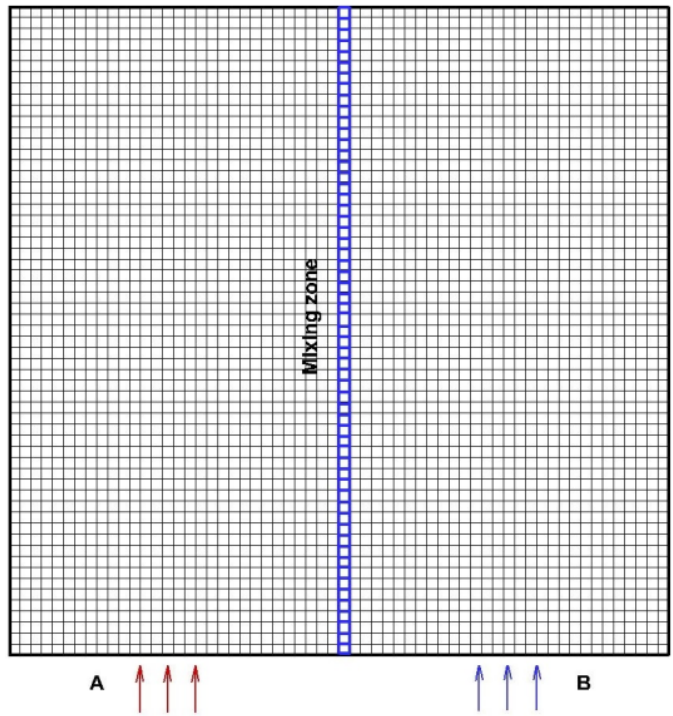

Figure 2 The computational domain used in the hybrid simulation. The dimension of the computational domain is $30.5 \mathrm{~cm} * 30 \mathrm{~cm}(61 * 60$ grids). The STOMP grid is denoted in black; cells highlighted in blue also define potential SPH subdomains. Solutes A and B are introduced at the bottom of the domain at a uniform inlet flux rate, each solute on one half of the inlet boundary, thus forming a mixing and reaction zone along the central column of STOMP grid cells. Lateral boundaries are no-flow.

An initial STOMP simulation is performed for flow only (no transport or reaction) to spin the model up to steady flow conditions. The restart capability of STOMP is subsequently used to perform a sequence of transport simulations of one time step each, as described in the model coupling section below. Initial conditions for the first transport time step are zero concentration of all species over the model domain; solutes A and B are introduced at the inflow boundary (bottom of the domain) by continuously injecting concentrations of $\left(\mathrm{A}=A_{0} ; \mathrm{B}=0 ; \mathrm{C}=0\right)$ over the left half of the inlet boundary (grid cells $i=1,30)$ and $\left(\mathrm{A}=0 ; \mathrm{B}=B_{0} ; \mathrm{C}=0\right)$ over the right half $(i=32-61)$, where $A_{0}$ and $B_{0}$ are constant input concentrations. The two species mix and react along the domain centerline to form the reaction product $\mathrm{C}$ as all three species are transported upward through the domain. The STOMP grid is set up such that the mixing occurs primarily within one vertical column of grid cells at the center of the domain (that is, there are an odd number of grid cells in the horizontal axis direction). At the center cell on the bottom boundary ( $i=31$, $j=1$ ), A and B are each injected over half the boundary in the pore-scale simulations, but in the STOMP simulation this is approximated by injecting a concentration of $\left(\mathrm{A}=A_{0} / 2 ; \mathrm{B}=B_{0} / 2 ; \mathrm{C}=0\right)$ representing the average inlet concentration in that cell.

\subsection{Hybrid multiscale coupling of pore- and continuum-scale models}

At the resolution of the STOMP model grid $(0.5 \mathrm{~cm})$, it is not valid to assume complete mixing (uniform concentrations at the sub-grid scale) of the reacting species A and B in grid cells that lie along the mixing interface (central column of grid cells). Therefore, the effective reaction rate at the continuum scale will depend not only on the fundamental reaction rate coefficient (the fully mixed value used at the pore scale) but also on the specific spatial configuration of reactants A and B at the sub-grid (pore) scale. Of course, this issue could be avoided entirely by simulating the entire model domain at the pore scale, but to do so would not only be computationally prohibitive, but also wasteful as much of the model domain contains only A or B (and not both) at any given time, and the mixingcontrolled reaction occurs only in a small subdomain (principally along the center vertical column of STOMP grid cells). To maintain the accuracy associated with direct pore-scale simulation, while also taking advantage of the computational efficiency of the continuum-scale model where appropriate, we utilize a hybrid approach in which pore- and continuum-scale models are directly coupled together in a single simulation. In the language of multiscale mathematics [77], our approach is hierarchical in the sense that the continuum simulation is performed over the complete domain while pore-scale simulation is performed only on a limited subdomain, resulting in complete overlap between the two wherever pore-scale simulation is performed. This eliminates the need for strictly matching boundary conditions (e.g., fluid and solute mass fluxes) between the pore- and continuum-scale domains 
that would be otherwise necessary in a concurrent approach. The conceptual process of multiscale coupling used here can be described in the following steps:

1. Identify sub-regions in the continuum domain where mixing is likely to be incomplete (i.e., where porescale simulation is deemed necessary)

2. Initialize pore-scale simulations on the selected sub-regions based on the current macroscopic conditions.

3. Perform independent pore-scale simulations on each continuum-scale grid cell identified in step 1 for a specified time period, and use the results to compute spatially variable effective reaction rate coefficients to pass to the continuum scale model.

4. Advance the continuum-scale model in time using the modified reaction rate coefficients where porescale simulations were performed in step 2 and the constant (fully mixed) reaction rate coefficient everywhere else.

5. Based on the continuum-scale simulation results, return to step 1 and repeat the process until the full simulation time has elapsed.

The details of the implementation of this general approach are provided in the sections below.

\subsubsection{Dimension Reduction with Numerical Closure}

The approach used to couple the pore- and continuum-scale models follows the general methodology outlined and demonstrated in [71], which they refer to as Dimension Reduction with Numerical Closure (DRNC). In any rigorous upscaling methodology, one first averages microscale equations and then applies one or more scaling laws (closure approximations) that allow elimination of microscale variables from the averaged macroscopic equations [78]. If an appropriate set of scaling laws cannot be identified, the microscale variables remain embedded in the macroscale equation in the form of volume integrals (non-local terms). Here, because we do not know and cannot generally assume a form for the microscale distribution of reactants A and B within the sharp mixing interface regions, we posit that a general closure is not appropriate. However, it is possible nonetheless to perform a numerical closure by directly computing the microscale variables (in this case, the pore-scale distribution of $\mathrm{A}$ and B) and computing the non-local terms directly from the microscale variables. Since these distributions change over time, it is necessary to iteratively update the numerical closure as the simulation progresses. This requires repeatedly going back and forth between the macroscale and microscale model in the affected sub-regions, which in turn requires a method for upscaling microscale variables to the macroscale (called restriction, e.g., [79]) and a method for re-initializing microscale simulations after a period of macroscale simulation (called lifting or reconstruction). The latter is usually much more difficult, as the microscale reconstruction from macroscale variables is often highly non-unique. In some cases where the reconstruction can be performed very accurately, it is not actually necessary to perform microscale simulation, as the new macroscale parameters can be derived directly from the reconstructed microscale variables (e.g., [80]). In other cases (e.g., [71]), the reconstruction is inaccurate but the microscale system relaxes quickly (loses memory of initial conditions) relative to time scales of macroscale dynamics, such that only a short burst of microscale simulation is necessary. In the work reported here, we try to minimize error in the reconstruction by performing pore-scale simulation over a period of time equivalent to the macroscopic time step, such that there is no gap in time between the restriction and reconstruction operators. Our approach gains computational efficiency (relative to complete pore-scale simulation) primarily by limiting the spatial extent of pore-scale simulations within the overall model domain; additional computational efficiency might be gained by reducing the time of individual microscopic simulations. Future work will explore the effect of reducing pore-scale simulation times on overall model predictions through introduction of error in the reconstruction process to determine the tradeoff between model accuracy and computational efficiency. Our approach follows the general pattern of the heterogeneous multiscale method as described by [81] but has unique implementation details. It is also similar to the equation-free method (EFM; [79]), but differs significantly in that we utilize formal partial differential equations at both the microscale and macroscale, where the EFM does not specify macroscale equations but drives the macroscale system directly from microscale simulations. 
For the purposes of this study, most macroscopic parameters (e.g., hydraulic conductivity, dispersivity, porosity) are assumed to be well-defined and do not depend explicitly on microscale variables. We focus primarily on the reaction rate coefficient, as this macroscopic parameter is likely to be highly dependent on the specific configuration of microscale concentrations at any point in space and time, particularly in the vicinity of a sharp mixing front. Our restriction operator is straightforward, and can be derived directly by comparing the average of microscale reaction rates to the average macroscale reaction rate. As noted above, the macroscopic (continuum-scale) reaction rate for a given grid cell is given by:

$$
R_{\text {cont }}=\frac{d[C]}{d t}=k_{f, e f f}[A][B]
$$

At the pore scale, the corresponding reaction rate is defined for each computational particle $i$ as:

$$
R_{\text {pore }}=\frac{d\left[C_{i}\right]}{d t}=k_{f}\left[A_{i}\right]\left[B_{i}\right]
$$

The spatially-averaged reaction rate over the pore-scale domain (corresponding to a single STOMP grid cell) is:

$$
\frac{1}{N_{p}} \sum_{i=1}^{N_{p}} \frac{d\left[C_{i}\right]}{d t}=\frac{1}{N_{p}} \sum_{i=1}^{N_{p}} k_{f}\left[A_{i}\right]\left[B_{i}\right]
$$

which reduces to

$$
\frac{d \bar{C}}{d t}=\frac{k_{f}}{N_{p}} \sum_{i=1}^{N_{p}}\left[A_{i} B_{i}\right]
$$

In which $\bar{C}$ is the macroscopic (average) concentration over the pore-scale domain (applicable to the corresponding macroscale grid cell). On the right hand side, however, the applicable average is that of the product of $\mathrm{A}$ and $\mathrm{B}$, not the product of the averages of $\mathrm{A}$ and $\mathrm{B}$. That is:

$$
\frac{d \bar{C}}{d t}=k_{f} \overline{A B}
$$

Therefore, it cannot be written directly in terms of the macroscopic concentrations of A and B. We introduce a mixing factor $m$ which is the ratio of the average of the product of concentrations to the product of average concentrations:

$$
m=\frac{\overline{A B}}{\bar{A} \bar{B}}=N_{p} \frac{\sum_{i=1}^{N_{p}} A_{i} B_{i}}{\sum_{i=1}^{N p} A_{i} \sum_{i=1}^{N p} B_{i}}
$$

which allows us to write the rate formulation as

$$
\frac{d \bar{C}}{d t}=k_{f} m \bar{A} \bar{B}
$$

Now the equation is written entirely in terms of average concentrations as described by the macroscopic model. This form can be used over the entire macroscopic domain. In regions away from the mixing interface, where there is complete mixing within macroscopic grid cells, $m=1$ (that is, the standard reaction form holds at the macroscale). In those macroscale grid elements corresponding to the microscale domains, $m$ must be calculated from the results of the pore-scale simulation, and updated periodically using the dimension reduction approach to maintain accuracy. 


\subsubsection{Reconstruction (Lifting)}

Because we perform both microscopic and macroscopic simulations over the same time period at those regions where there is overlap (regions where pore-scale simulation is performed), the need for reconstruction is minimized. The primary constraint is that we must ensure that the total mass of reactants (A and B) and reaction product (C) is consistent. That is, the total mass of each solute summed over the pore-scale discrete elements (SPH particles) must equal the macroscopic concentration in the corresponding grid cell multiplied by the cell volume. This is accomplished through a simple rescaling of the SPH particle solute masses to match the total mass from the macroscale simulation after each macroscale time step (before an episode of pore-scale simulation):

$$
I_{i}\left(\Delta t N_{r}+\Delta T\right)=\frac{\langle I\rangle\left(\Delta t N_{r}+\Delta T\right)}{\sum_{j \in f l u i d} I_{j}\left(\Delta t N_{r}\right)} I_{i}\left(\Delta t N_{r}\right)
$$

where $I_{i}$ is the concentration of solute $I(I=A$ or $B)$ of fluid particle $i,\langle I\rangle$ is the macroscopic average concentration of solute $I(A$ or $B)$, and other terms are as defined above. Therefore the spatial distribution of pore-scale concentrations (and the associated mixing coefficient $m$ ) is retained but the actual magnitudes of SPH particle concentrations may be slightly adjusted. More details of the reconstruction method can be found in [71].

\subsubsection{Independence of pore-scale simulations}

The loose coupling approach used here facilitates computational efficiency by allowing each pore-scale simulation (which is the most computationally intensive portion of the workflow) to run independently of other porescale simulations. That is, they are executed as "task-parallel" [82, 83], which provides us with two levels of parallelism that can be exploited for computational speedup. Each individual pore-scale simulation uses a parallel code (based on domain decomposition) and can be executed on many processors, and since each pore-scale simulation is independent of the others they can be run simultaneously without inter-processor communication. Therefore, if we are running pore-scale simulations on $N_{d}$ different macroscale subdomains during a particular time step, and each pore-scale simulation can efficiently utilize $N_{p}$ processors, then the overall effort can be distributed efficiently to $N_{d} * N_{\mathrm{p}}$ processors with near-optimal speedup.

In fact, however, the pore-scale subdomains are not strictly independent, because they share adjacent boundaries through which fluid and solutes flow (see SPH cells in Figure 2). To decouple these boundary conditions and ensure independence of the pore-scale simulations, we use simulated concentrations at the top of one SPH domain (from the end of the prior simulation period) to specify influx concentrations in the current simulation period for the bottom boundary of the neighboring (above, downstream in the sense of flow direction) SPH domain. As described previously, any particles entering the inlet (bottom) boundary of an SPH domain are assigned concentrations of A and $\mathrm{B}$ according to a discrete distribution along the inlet boundary provided by an input file. The input file is constructed by averaging the SPH particles in the domain immediately below the element under consideration within the discrete bins and down to a specified depth. That is, only particles near the outflow boundary of the upstream domain are used to prescribe inlet concentrations for the downstream domain. The approximations inherent in this approach are: 1) particle concentrations are averaged within each bin along the interface (we use 20 bins in the simulations reported here), and 2) internal changes in particle concentrations occurring during one simulation period (one macroscale time step) are not reflected in the boundary fluxes until the next simulation period. 
The SPH code [72] was modified to support the boundary conditions unique to this problem. Normally, the SPH code utilizes periodic boundary conditions; particles leaving the system at one boundary are reintroduced at the opposing boundary. While this is appropriate for the flow solution, simulation of solute transport requires a method to appropriately assign solute concentrations to re-entering particles depending on which boundary they are entering through. For the inflow (bottom) boundary, the SPH code was modified to read an input file comprised of discrete bins each with specified concentrations of reactants $\mathrm{A}$ and $\mathrm{B}$ and reaction product $\mathrm{C}$, and apply those concentrations to any particles entering that boundary during the associated simulation period. For the lateral boundaries, there are no corresponding microscale (SPH) domains adjacent to a particular SPH domain. Therefore, particles exiting the domain on one side and re-entering on the other were assigned new concentrations of $\mathrm{A}, \mathrm{B}$, and $\mathrm{C}$ based on linear interpolation of concentrations in the adjacent STOMP grid cells from the previous STOMP time step.

\subsubsection{Adaptive selection of pore-scale domains}

After each macroscale time step, it is necessary to select those macroscale grid cells within which microscale (porescale) simulation will be explicitly performed during the next time step. This decision must be made on the basis of macroscale information, as microscale information is only available at limited locations and times. In general, a sophisticated approach to adaptive selection should employ generalized applicability criteria derived from a rigorous upscaling analysis as described in [67-69]. However, our simple example problem is designed such that it is evident a priori that the critical region for potential pore-scale simulations is the single column of grid cells denoted as SPH domains in Figure 2, where mixing of the two reactants occurs in this homogeneous system. Therefore, we utilize a simpler criterion in which pore-scale simulation is initiated when the product of average concentrations of A and B at the macroscale exceeds a very small user-specified value (that is, some low level of initial solute has entered the grid cell). The average concentration of the pore-scale domain is obtained from the macroscale simulator (STOMP) output. For any pore-scale domain ' $i$ ', we define a pore-scale domain to be active when,

$$
\frac{\left[A_{i}\right] \cdot\left[B_{i}\right]}{A_{\max } B_{\max }}>4.0 \times 10^{-6}
$$

where $\left[A_{i}\right]=$ average concentration of solute $\mathrm{A}$ in domain $\mathrm{i},\left[B_{i}\right]=$ average concentration of solute $\mathrm{B}$ in domain $\mathrm{i}$, and $\mathrm{A}_{\max }$ and $\mathrm{B}_{\max }$ are the maximum (inlet) concentrations of solutes $\mathrm{A}$ and $\mathrm{B}$, respectively. Upon activation, the concentration of all particles in the pore-scale simulation domain are initialized to the average concentrations from the macroscale grid cell; since these are initialized at very low concentrations, the spatial distribution at the pore scale does not have a significant impact. For the first time step, the number of pore-scale domains is $N_{p s}=1 . N_{p s}$ is then incremented each time one or more pore domains are activated. After some number of macroscale time steps (and corresponding microscale simulation periods), the solute front will have passed through a particular subdomain and the configuration of $\mathrm{A}, \mathrm{B}$ and $\mathrm{C}$ in that subdomain can be expected to reach a quasi-equilibrium, after which porescale simulation can be discontinued. In this work, we did not apply any criterion to discontinue pore-scale simulation because we wanted to observe the pore-scale behavior over the full simulation time period. However, it would be possible to reduce the pore-scale computational demand by turning off pore-scale simulation domains once a quasi-equilibrium state is reached. In this approach, the estimated mixing coefficient from a given pore-scale subdomain would be compared over a series of time steps to determine when it has stabilized (changes are within a specified bound) and subsequent time steps would use the stabilized value in macroscale simulation without performing updates through microscale simulation. This method would reduce the number of pore-scale simulation domains required at each time step of the hybrid simulation. 


\subsubsection{Hybrid workflow}

The hybrid task workflow is presented in Figure 3, which follows the conceptual model explained in Sec. 2.3 and consists of four main modules: 1) a serial version of the macroscale simulator (STOMP) covering the full computational domain, 2) a serial python script - Pore Generator (PG) - that adaptively determines pore-scale simulation regions, executes the reconstruction step, and constructs SPH input files, 3) parallel execution of porescale simulations using SPH, and 4) a serial python script - Grid Parameter Generator (GPG) - that performs the numerical closure and constructs STOMP input files. The STOMP simulator [73] is used to compute the macroscale reaction. Configuration files (including an initial mixing coefficient file) are provided to initiate the simulation. The Pore Generator (PG) provides algorithms to update pore-scale particle properties based on macroscale quantities from the previous STOMP time step. It consists of two sub-modules: 1) an Adaptivity Manager that determines how many and which pore-scale subdomains require simulation and 2) a Pore Concentration Generator which performs the reconstruction of the pore-scale initial conditions for pore-scale subdomains. The SPH code [72] is executed to perform pore-scale simulations for each active pore-scale subdomain determined by PG. The Grid Parameter Generator then creates updated STOMP input files (in particular the file containing mixing coefficient values) based on output from SPH runs. 

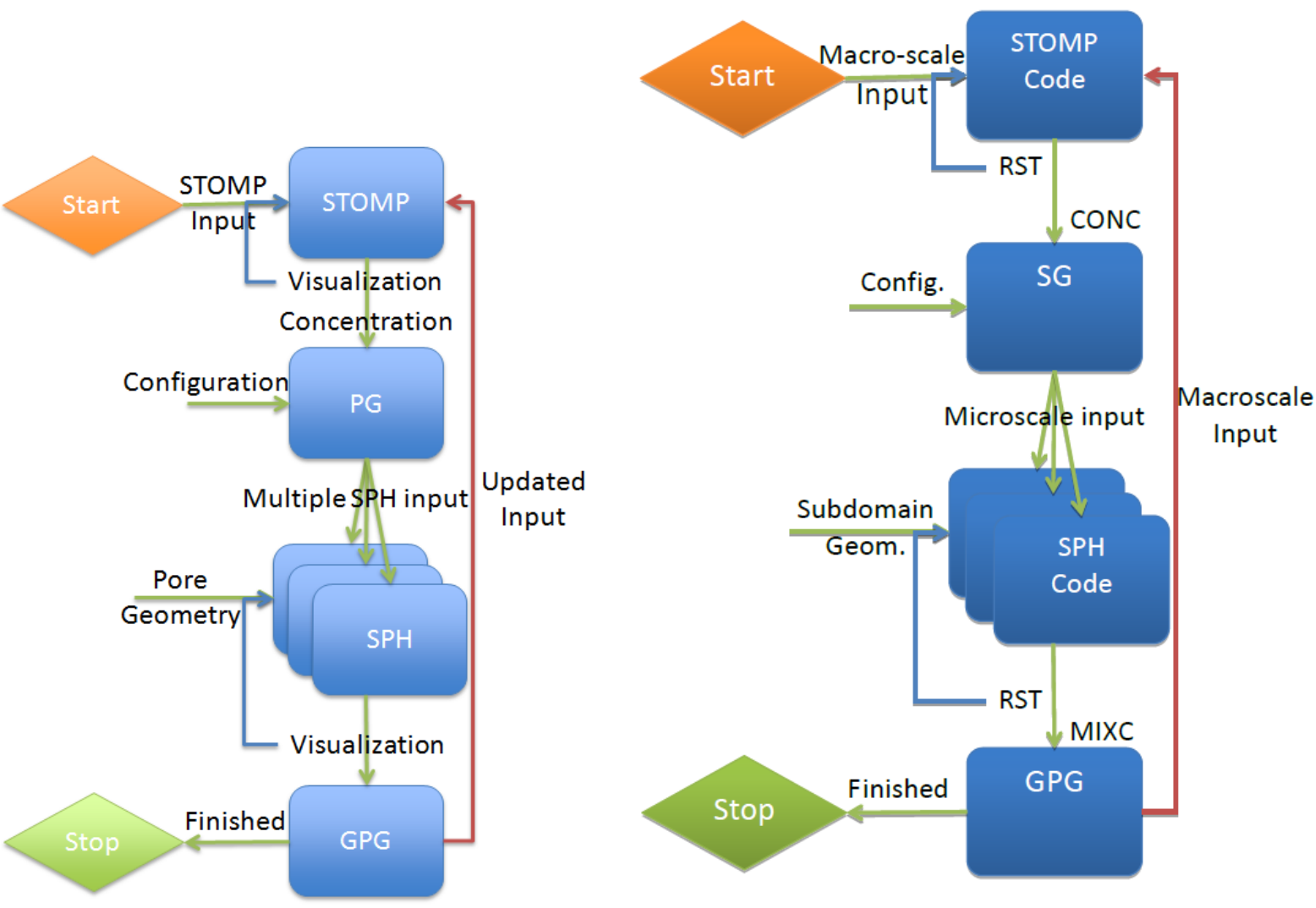

Figure 3 Workflow of the hybrid simulation

The hybrid model workflow is executed and managed using the Swift workflow environment [74]. It is launched by invoking a single instance of Swift, along with a request to allocate all the resources (computational nodes) anticipated to be needed during the entire course of the execution, which eliminates the need for each independent STOMP and SPH simulation to wait in the supercomputer queuing system multiple times. Swift provides the scripting environment needed to launch and monitor individual SPH and STOMP simulations and run the processing scripts within the allocated set of computational nodes. The workflow is adaptive and portable. It supports dynamic scheduling of tasks, and utilizes Swift's logging and error handling capabilities. Post-processing functions are also supported by the workflow including visualization and provenance tracking capabilities.

The hybrid workflow was configured, tested and executed on the NERSC Hopper system (Cray XE6) using 1536 nodes (with 24 processors/node). The STOMP simulations are relatively small and execute in only a few seconds; $85 \%$ of computing time is spent on SPH runs. Within each iteration, the workflow executes no more than $60 \mathrm{SPH}$ simulations in parallel (the actual number adaptively depends on number of activated SPH cells). The overall simulation requires approximately 800 workflow iterations and approximately 96 hours of wall clock time for the complete run. More details of the hybrid workflow can be found in [76, 84]. 


\section{3. Results}

2 In this section, results of the hybrid multiscale simulation are presented. To evaluate the results, we compare the 3 predictions of our hybrid multiscale simulation with a comparable single-scale simulation (macroscale, STOMP 4 only). Comparisons are presented in terms of visualized concentration fields (reactants and product), effective 5 macroscale reaction rates, and total mass of accumulated product over the course of the simulation period.

\subsection{Macroscopic Concentrations}

The macroscopic (STOMP) concentrations of reactants (A and B) and reaction product (C) at the end of the hybrid

8 multiscale simulation period are visualized in Figure 4 (a-c). During the execution of the workflow, the reactants

9 sweep through the domain from bottom to top, forming a thin zone of reaction product at the mixing interface between the two reactants in the middle of the domain. 


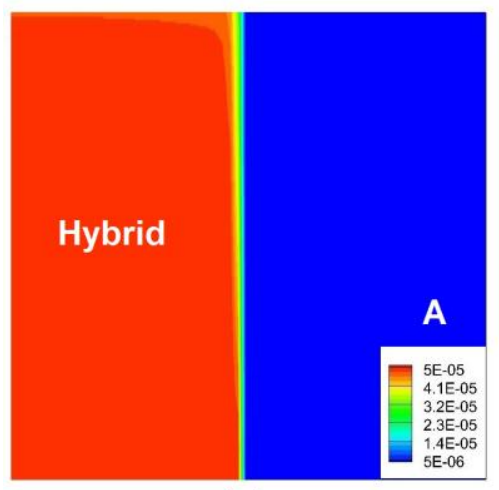

(a)

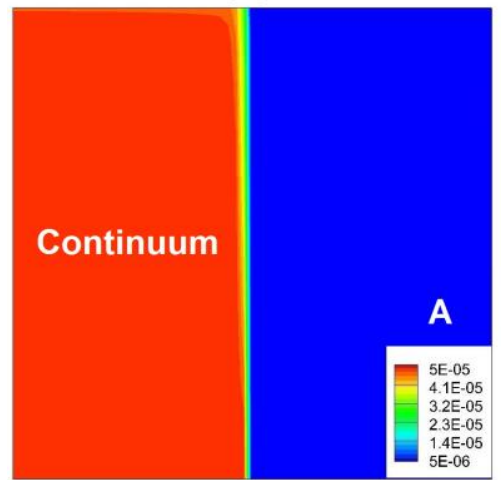

(d)

2

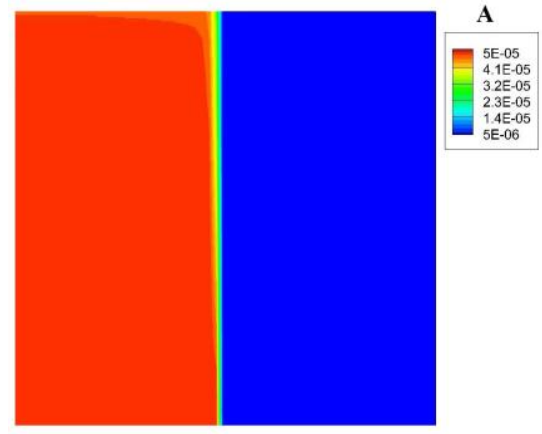

(a)

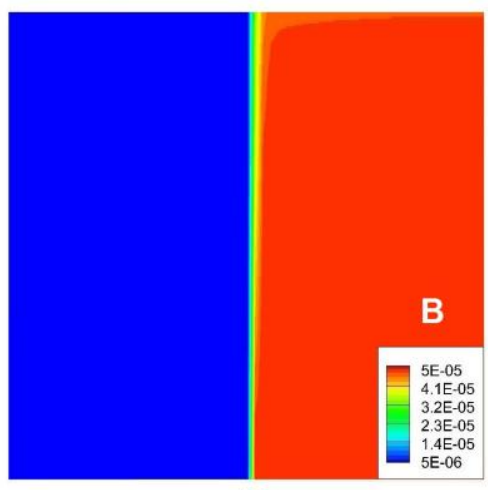

(b)

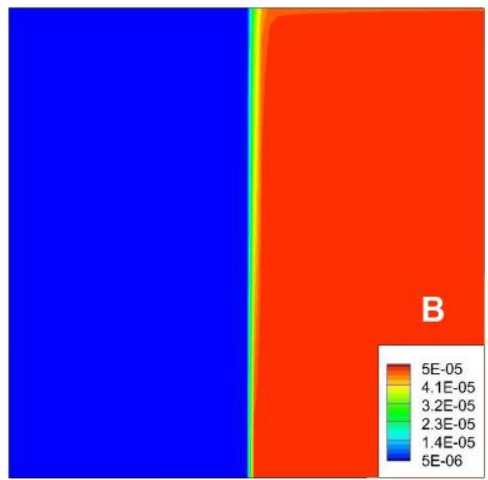

(e)

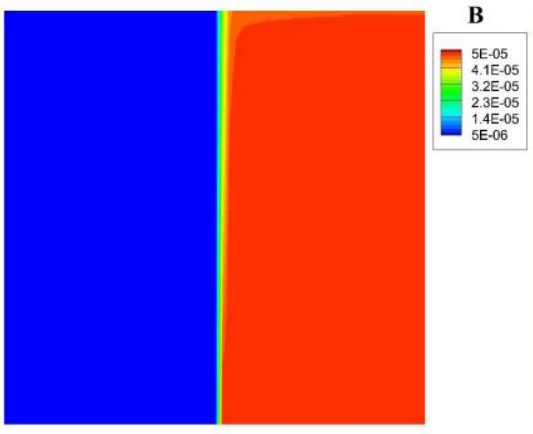

(b)

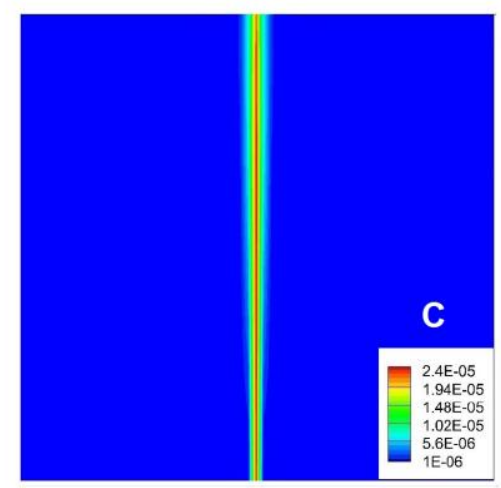

(c)

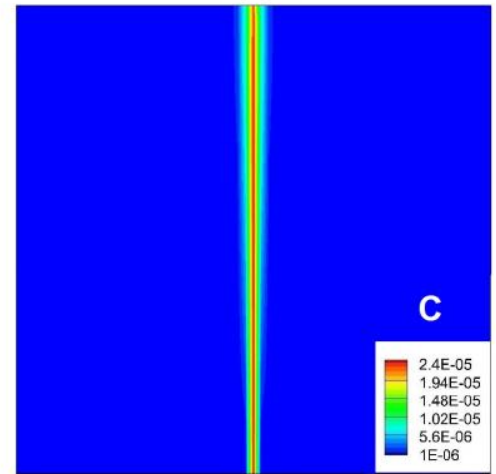

(f)

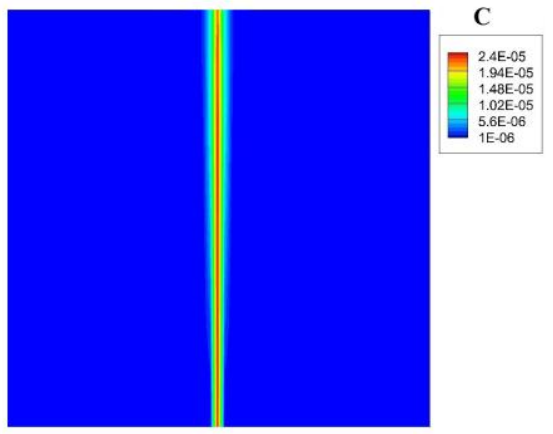

(c)

Figure 4 Top: Hybrid simulation (STOMP+SPH) : (a) concentration of reactant A; (b) concentration of reactant B; (c) concentration of product C Bottom: Single-scale (continuum-scale, STOMP only) simulation: (d) concentration of reactant A; (e) concentration of reactant B; (f) concentration of product $\mathrm{C}$. The units of the concentration are mols $/ \mathrm{cm}^{3}$. 
1 A corresponding single-scale simulation was performed using the STOMP model with the same initial and 2 boundary conditions, grid configuration, and macroscopic parameters as used in STOMP simulations for the hybrid 3 multiscale simulation. The only difference between the single-scale model and the hybrid multiscale model is that the single-scale model uses a constant reaction rate coefficient $\left(k_{f, e f f}\right.$ in Eqn. 3$)$ in all STOMP grid cells, whereas in the hybrid multiscale model the reaction rate coefficients in the center column of grid cells are updated at each time step based on the results of pore-scale simulations (through modification of $k_{f, e f f}$ by the mixing coefficient $m$ as in Eqn. 9).

7 Figure 5 (d-f) shows the results of the single-scale model (compare to Figure 4 (a-c)). The results obtained from the 8 single-scale simulation are slightly more diffusive in the mixing zone than the hybrid simulation results. Figure 5 
Advances in Water Resources

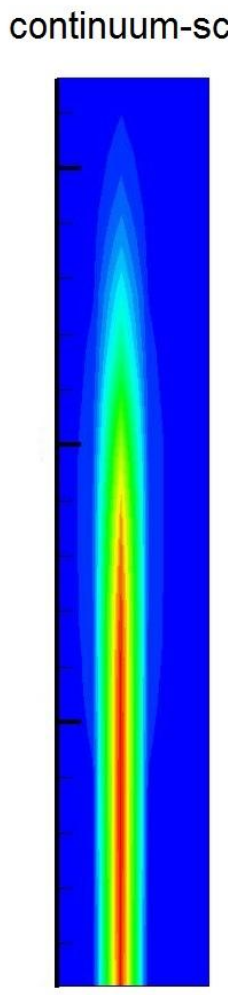

(a) hybrid multiscale

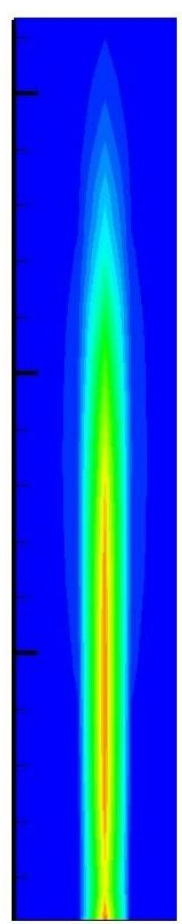

(b)

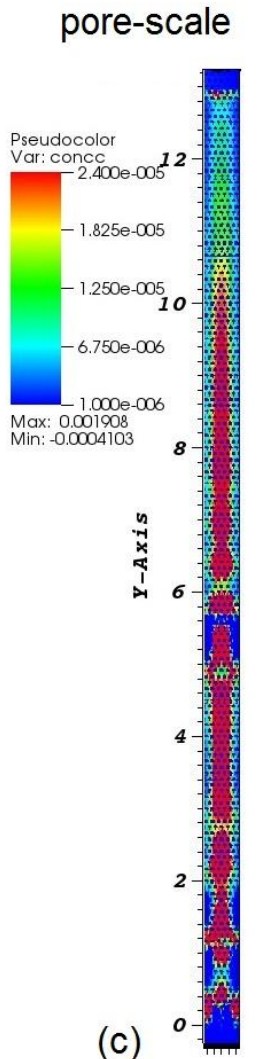

(c)
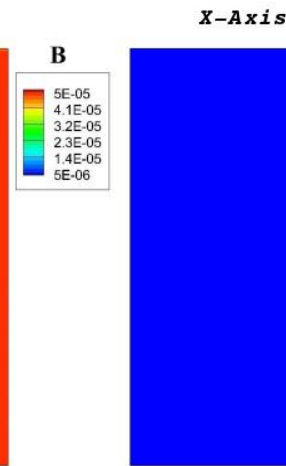

(b)

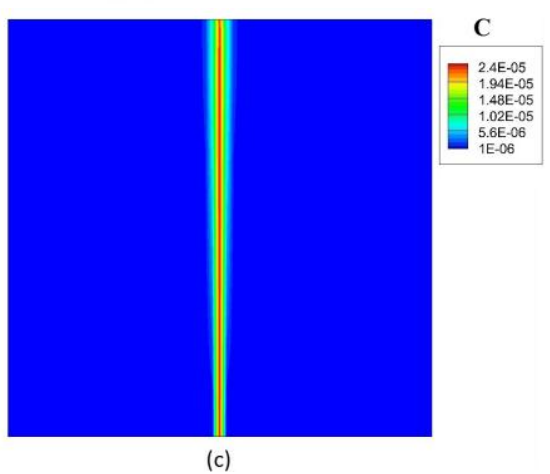

(c)

Figure 5 Concentration of product $\mathrm{C}\left(\mathrm{mols} / \mathrm{cm}^{3}\right)$ comparisons between single-scale (continuum-scale, STOMP only) simulation, hybrid multiscale simulation and pore-scale simulation $(\mathrm{SPH})$ at iteration \#327. 
Advances in Water Resources

\subsection{Pore-scale Concentrations}

Figure 6 shows visualizations of both macroscale (STOMP) and pore-scale (SPH) reaction product concentrations. For pore-scale domains, concentrations of reactants A and B are also shown. For purposes of visualization, all active pore-scale subdomains are stacked to create a single pore-scale image corresponding to a portion of the macroscopic domain. Four snapshots at different selected times of the hybrid simulation are presented. At the pore scale, some artifacts of the boundary conditions imposed on pore-scale subdomains to break interdomain dependencies are evident, particularly at early times (time step 50). In particular, the assumed lateral boundary conditions (which derives concentrations assigned to entering SPH particles by interpolating STOMP concentrations) introduces some spurious solute into the sides of the pore-scale domains which creates artifacts that are then propagated upward. However, these artifacts do not persist as the front progresses through the system; by later times (time step 400), the microscopic distribution of reaction product $\mathrm{C}$ is fairly smooth and exhibits artifacts only near the inlet boundary. The artifacts can be reduced somewhat be reducing the macroscopic time step, but the improvement in solution is small compared to the additional simulation cost. In future work, we will test alternative boundary condition assumptions (such as deriving concentrations for SPH particles entering on lateral boundaries from the associated particle leaving on the opposite side) to see if these artifacts can be more easily minimized. 


\section{Product C}

Concentration $\left(\mathrm{mols} / \mathrm{cm}^{3}\right)$

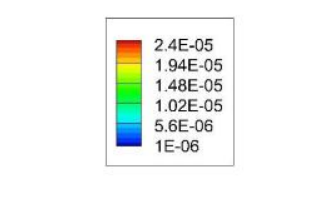

50

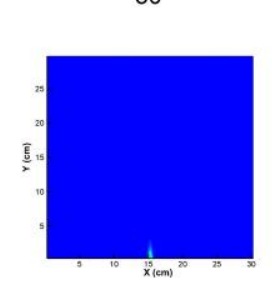

4 moments at iteration $\# 50, \# 250, \# 400, \# 600$

At each moment:

Left - mixing zone output from continuum-scale simulation (STOMP)

Right - mixing zone output from pore-scale simulation (SPH)

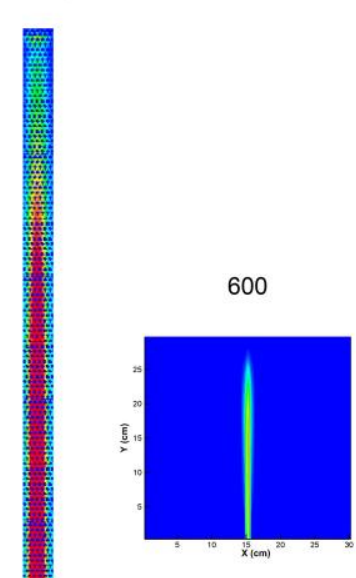


Advances in Water Resources

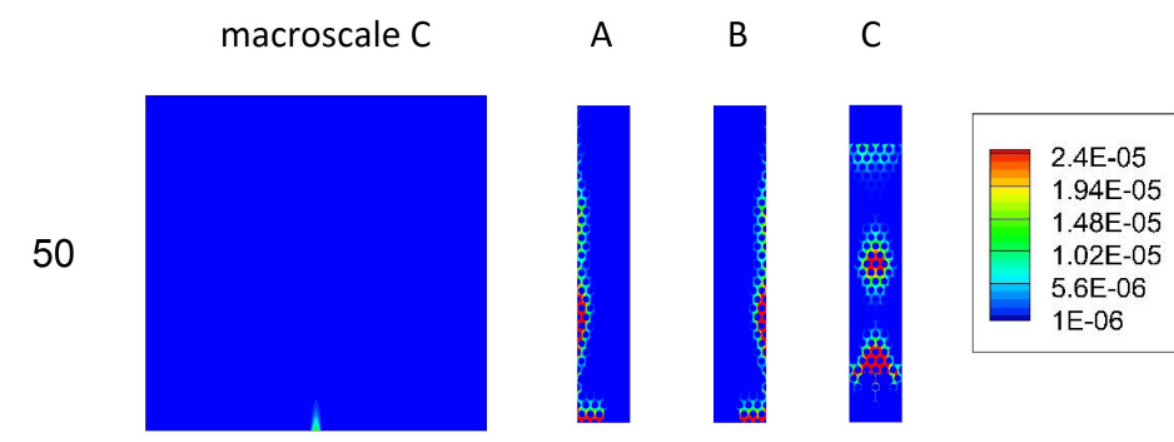

250
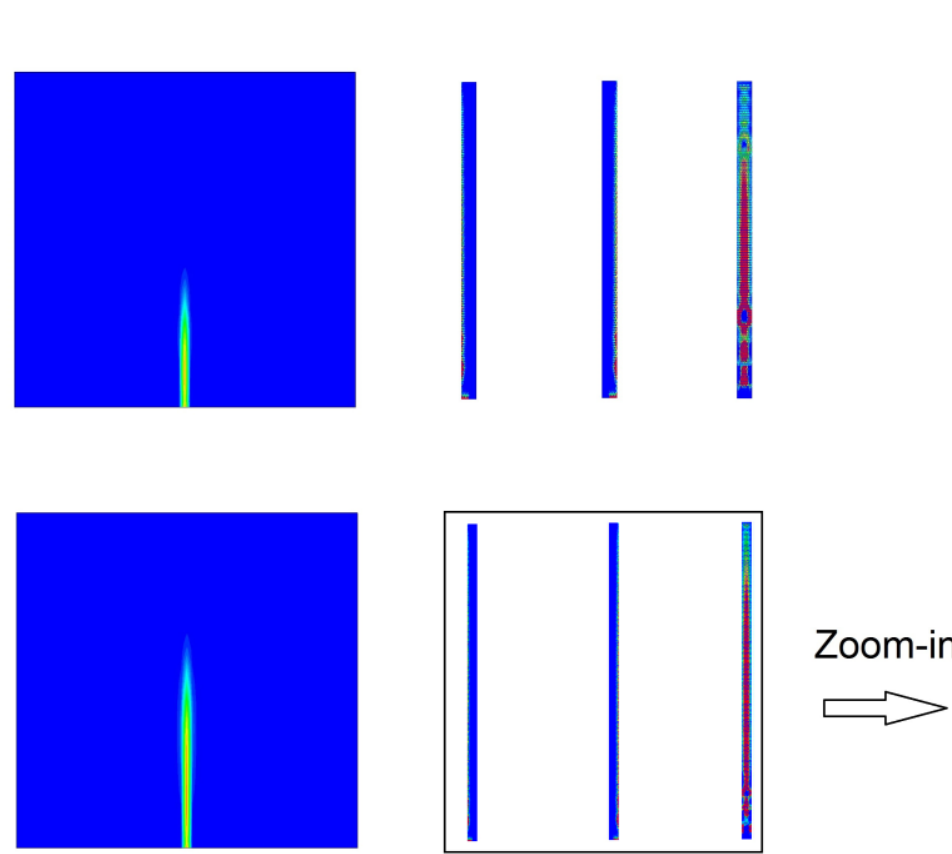

400
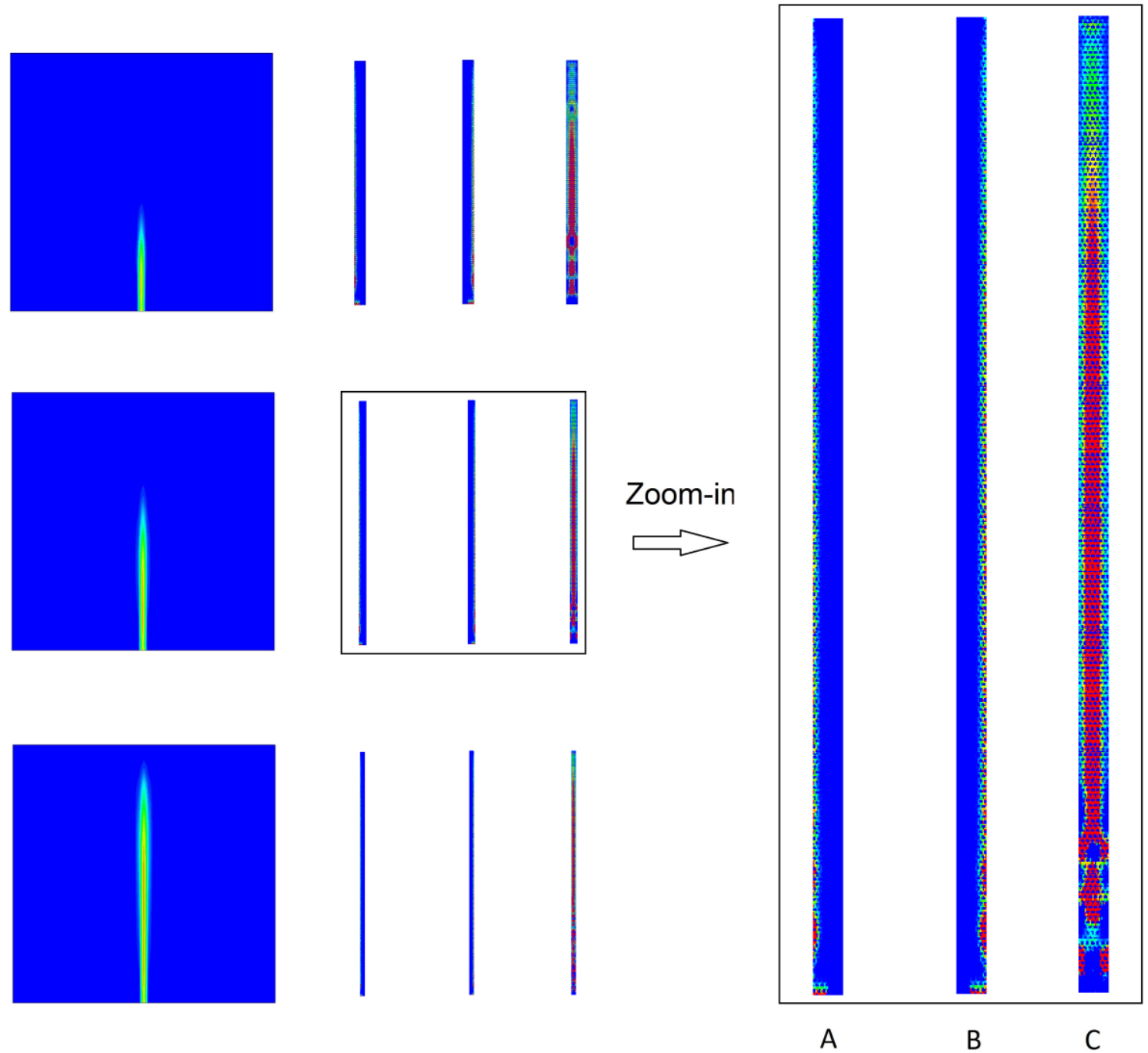

A

C 
Advances in Water Resources

1

Figure 6 Concentration comparisons between macroscale and pore-scale simulations in the hybrid workflow: the contour plots for product $\mathrm{C}$ at macroscale were outputs from STOMP simulations; the contour plots for reactants A, B and product C at microscale were stacked outputs from SPH simulations in activated subdomains.

\subsection{Reaction rates and reaction product mass}

The time evolution of local reaction rates (derived from the pore-scale simulation) is presented in two ways. Figure 7 shows visualized images of the spatial distribution of local reaction rates over time. That figure shows that mixing limitations at the pore scale reduce the effective reaction rate at the macroscale significantly along the vertical mixing interface, and that those reduced rates propagate upward through the system as the simulation proceeds. To provide additional detail, Figure 8 shows time series plots of local reaction rates for a small number of selected SPH subdomains. Two characteristic behaviors are indicated. At $\mathrm{j}=1$ (the cell immediately adjacent to the inflow boundary), the effective reaction rate quickly increases to a small value of approximately 0.01 (compare to nominal value of 0.35 ) at which it stabilizes for the remainder of the simulation (Figure 8a). At cells farther away from the inlet boundary, the effective reaction rate is set at the nominal (fully mixed) value of 0.35 until a pore-scale simulation is activated (when solutes reach the subject cell). At that time, the effective reaction rate drops rapidly and stabilizes at a very small value (approximately 0.0003) after some initial instability (oscillations). 
Advances in Water Resources
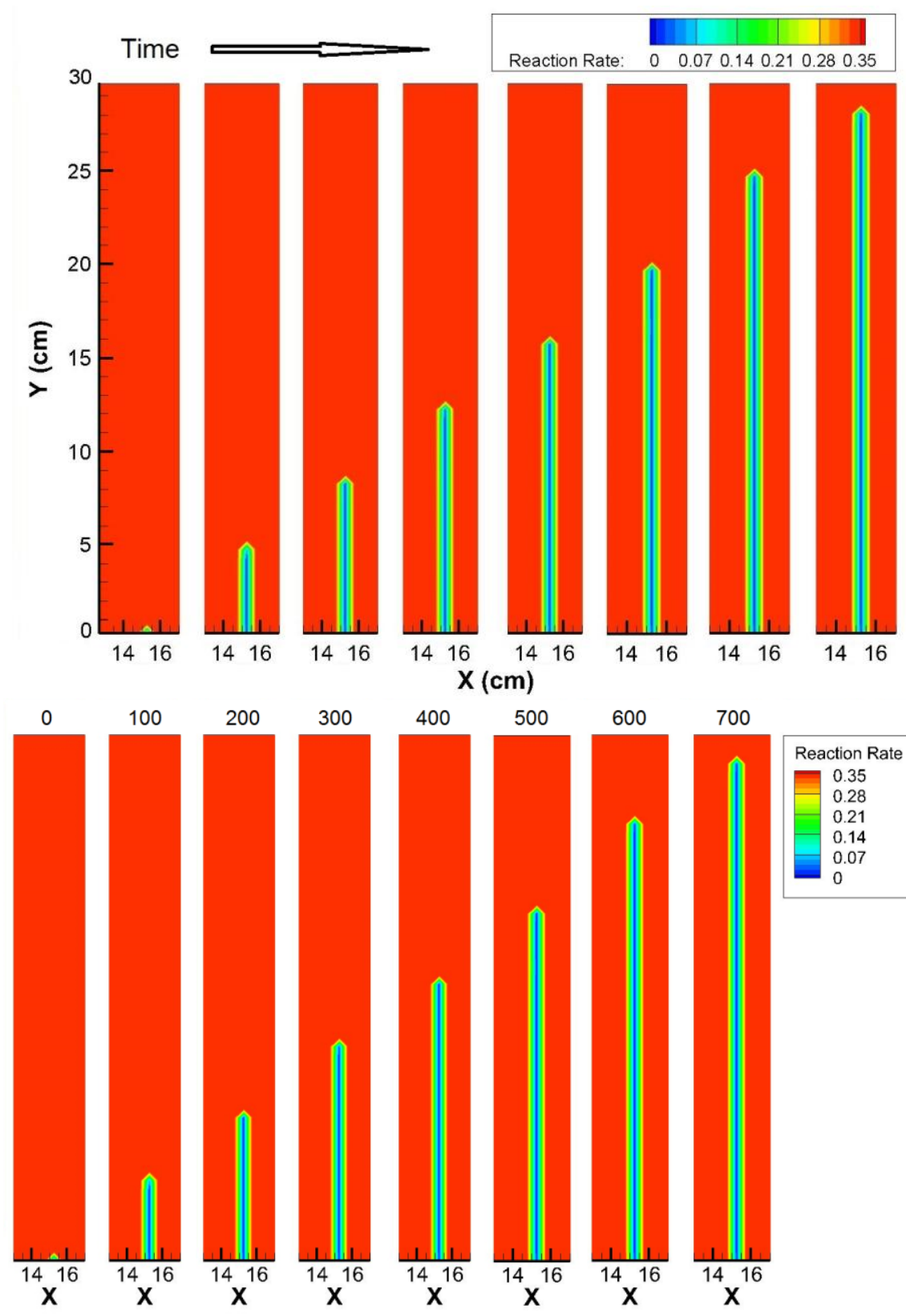

600
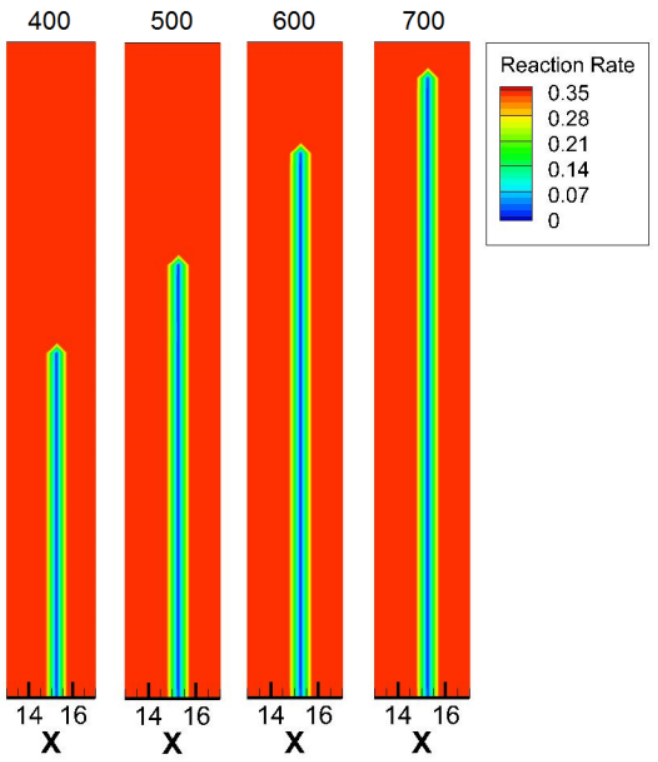

Figure 7 Time evolution of the local reaction rate during hybrid simulation (time step number is indicated by numerals at the top of each image). Only the central portion of the domain is shown for clarity. 


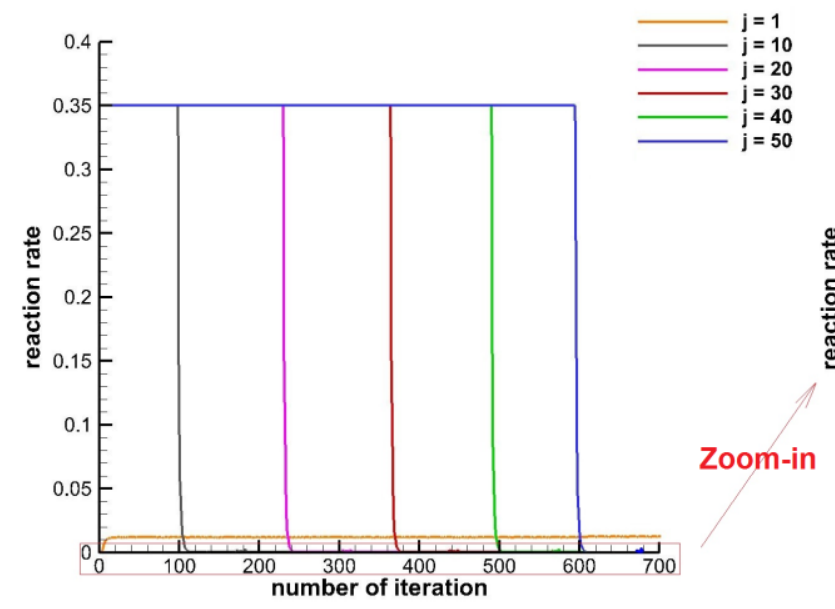

(a)

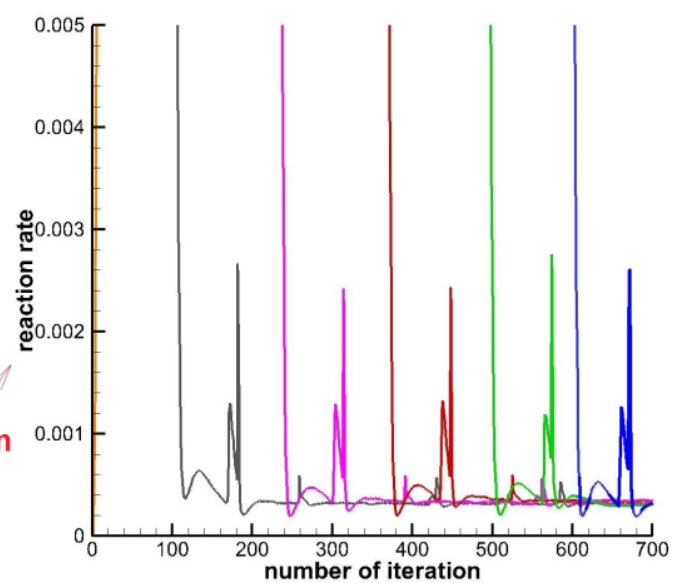

(b)

Figure 8 Time histories of local reaction rate at six locations (six SPH subdomains) along the central column of the computational domain: (a) normal view; (b) reduced vertical axis scale. Index j denotes the vertical index of the STOMP grid cell corresponding to the local SPH subdomain; $\mathrm{j}=1$ is the bottom-most cell and $\mathrm{j}=50$ is close to the top of the domain.

Because of the finely-resolved macroscopic grid and the small value of transverse dispersivity employed in the STOMP simulations, the visual differences between the single-scale and the hybrid simulations in terms of reaction product generated are minimal (Figure 4 and Figure 5). However, the effect of the modified reaction rate can better be observed by evaluating the total mass of reaction product generated. A comparison of time histories of the total mass of reaction product (C) between single-scale and hybrid models is presented in Figure 9. The total mass of reaction product $(\mathrm{C})$ at the end of the simulation period is $0.00215 \mathrm{~mol} / \mathrm{ml}$ in the single-scale model and 0.00187 $\mathrm{mol} / \mathrm{ml}$ in the hybrid multiscale. Because of the incorrect assumption of complete mixing at the grid scale in the single-scale model, the effective rate of reaction is too high and the amount of reaction product generated is overestimated by $\sim 15 \%$ percent. While we do not have experimental data that can be used to quantitatively test this result, it is qualitatively consistent with a large body of literature that has demonstrated reduced effective reaction rates in mixing-controlled reaction systems (e.g., $[10,47,50,85]$ ) It is interesting that the differences in produced mass are relatively small while the local reaction rate is diminished by orders of magnitude in the central cells. We attribute this fact to the effect of a "dead zone" that sets up at both model scales during the reaction of the two propagating solute bodies. As the reactants (A and B) move upward, they react at the centerline thus diminishing the concentration of $\mathrm{A}$ and $\mathrm{B}$ and increasing that of $\mathrm{C}$ in a zone encompassing the centerline. In this zone, because there are low concentrations of reactants, and their replenishment is limited by very slow transverse diffusion (microscale) and dispersion (macroscale), it becomes effectively a "dead zone" for reaction. The width of this zone is larger at the macroscale than at the microscale but in both cases the reactant concentrations approach zero. Since the rate is the product of the reactant concentrations and the reaction rate coefficient, the reaction rate in both dead zones approaches zero regardles of the actual value of the reaction rate coefficient. Thus the primary impact of pore-scale processes is exerted prior to the setup of these "dead zones", as reflected in the fact that the differences in product 
masses generated are greatest near the beginning of the solution and then the production rates become similar (lines in Figure 9 become parallel) after a few hundred iterations.

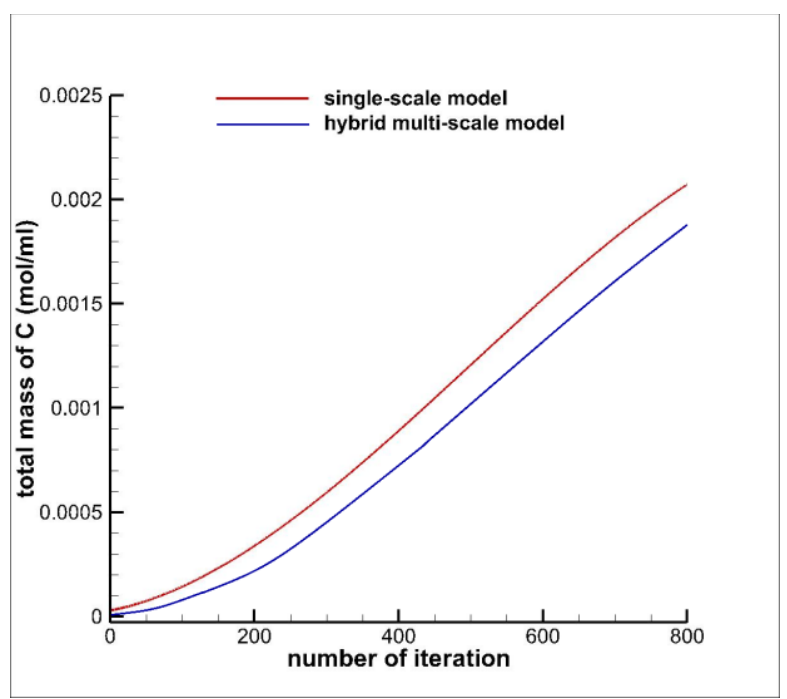

Figure 9 Time histories of the total mass of reaction product $\mathrm{C}$ in the single-scale and hybrid simulations.

We note that had we continued the simulations for a longer time period, both curves in Figure 9 would eventually flatten as the system approached a quasi-steady state in which the product mass flux out of the domain through the upstream boundary equals the rate of product mass generation by the reaction within the domain.

\section{Conclusions}

We have demonstrated a hybrid multiscale approach to coupling pore- and continuum-scale porous media flow and reactive transport simulators for a physical system with practical application. Our loosely-coupled simulation approach provided an efficient and feasible way to properly account for incomplete mixing at the sub-grid scale, and results in a $15 \%$ reduction in the total mass of reaction product generated in our simple test case. We note that while the complexity of the hybrid multiscale approach may limit its widespread application in practice, its use in test cases may provide insights that would improve the quality of less complex models. For example, having performed the hybrid simulation herein, we hypothesize that the observed $15 \%$ reduction in total product mass could be accurately simulated by performing a limited pore-scale or hybrid simulation to determine the appropriate value asymptotic pore-scale mixing coefficient, then simply applying that value in the continuum model wherever a sharp gradient in macroscopic concentrations exists. Alternatively, new upscaling approaches could be supported by hybrid model results; in this case, volume averaging using a jump condition to represent the sharp mixing interface (e.g., [86]) may be appropriate and could be tested using the hybrid solution.

The example problem considered here is quite simple, and was selected as an initial case for testing our novel hybrid multiscale approach. Further development and testing is needed in several areas to increase the applicability of 
this method to more realistic problems. Future research will focus on the following aspects of the method: 1) application to a more complex reaction system with a precipitated mineral phase, which is expected to increase the impact of imperfect mixing on the reaction; 2) application to heterogeneous porous media in which the location of the mixing zone is not explicitly known a priori, therefore requiring improvements in the adaptivity routines; 3 ) design and implemention of one or more experiments to test the model approach; 4) generalization to problems without a single localized mixing region; and 5) work to increase the computational efficiency of the method.

While computational power is increasing significantly and our understanding of biological and environmental processes at fundamental scales is accelerating, using this information to advance our knowledge of the larger system behavior requires the development of multiscale simulators. Accordingly there has been much recent interest in novel multiscale methods in which microscale and macroscale models are explicitly coupled in a single hybrid multiscale simulation.A limited number of hybrid multiscale simulations have been developed for biogeochemical earth systems, but they mostly utilize application-specific and sometimes ad-hoc approaches for model coupling. We are developing a generalized approach to hierarchical model coupling designed for high-performance computational systems, based on the current multiscale workflow framework. Future applications include simulation of biogeochemical impacts of groundwater - river water interactions coupling fine- and coarse-grid model representations [87].

\section{Acknowledgments}

This research was supported by the U. S. Department of Energy (DOE) office of Biological and Environmental Research through the PNNL Subsurface Science Scientific Focus Area project and the DOE office of Advanced Scientific Computing Research under the Scientific Discovery through Advanced Computing (SciDAC) program. Computations described here were performed using computational facilities of the National Energy Research Scientific Computing Center (NERSC), a national scientific user facility sponsored by DOE Office of Science. PNNL is operated for the DOE by Battelle Memorial Institute under Contract No. DE-AC06-76RLO 1830.

\section{References}

[1] Kang QJ, PC Lichtner, DX Zhang. An improved lattice Boltzmann model for multicomponent reactive transport in porous media at the pore scale. Water Resour Res. 43 (2007), doi: W12s14

$10.1029 / 2006$ wr005551.

[2] Kang QJ, PC Lichtner, DR Janecky. Lattice Boltzmann Method for Reacting Flows in Porous Media. Advances in Applied Mathematics and Mechanics. 2 (2010) 545-63, doi: 10.4208/aamm.10-10S02.

[3] Kang QJ, PC Lichtner, HS Viswanathan, AI Abdel-Fattah. Pore Scale Modeling of Reactive Transport Involved in Geologic CO2 Sequestration. Transport Porous Med. 82 (2010) 197-213, doi: 10.1007/s11242-009-9443-9.

[4] Van Leemput P, C Vandekerckhove, W Vanroose, D Roose. Accuracy of hybrid lattice boltzmann/finite difference schemes for reactiondiffusion systems. Multiscale Modeling \& Simulation. 6 (2007) 838-57, doi: 10.1137/060675113.

[5] Sukop MC, H Huang, CL Lin, MD Deo, K Oh, JD Miller. Distribution of multiphase fluids in porous media: Comparison between lattice Boltzmann modeling and micro-X-ray tomography. Physical Review E. 77 (2008), doi: 026710 10.1103/PhysRevE.77.026710.

[6] Huang HB, XY Lu, MC Sukop. Numerical study of lattice Boltzmann methods for a convection-diffusion equation coupled with Navier-Stokes equations. Journal of Physics a-Mathematical and Theoretical. 44 (2011), doi: 055001

10.1088/1751-8113/44/5/055001. 
Advances in Water Resources

1 [7] Maier RS, DM Kroll, RS Bernard, SE Howington, JF Peters, HT Davis. Hydrodynamic dispersion in confined packed beds. Phys Fluids. 15 2 (2003) 3795-815, doi: 10.1063/1.1624836.

3 [8] Maier RS, DM Kroll, RS Bernard, SE Howington, JF Peters, HT Davis. Pore-scale simulation of dispersion. Phys Fluids. 12 (2000) 2065-79, 4 doi: $10.1063 / 1.870452$.

5 [9] Tartakovsky AM, GD Tartakovsky, TD Scheibe. Effects of incomplete mixing on multicomponent reactive transport. Adv Water Resour. 32 6 (2009) 1674-9, doi: 10.1016/j.advwatres.2009.08.012.

7 [10] Tartakovsky AM, G Redden, PC Lichtner, TD Scheibe, P Meakin. Mixing-induced precipitation: Experimental study and multiscale 8 numerical analysis. Water Resour Res. 44 (2008).

9 [11] Tartakovsky AM, TD Scheibe, P Meakin. Pore-Scale Model for Reactive Transport and Biomass Growth. Journal of Porous Media. 12 (2009)

$10 \quad 417-34$

11 [12] Tartakovsky AM, P Meakin, TD Scheibe, RME West. Simulations of reactive transport and precipitation with smoothed particle

12 hydrodynamics. J Comput Phys. 222 (2007) 654-72.

13 [13] Tartakovsky AM, P Meakin, TD Scheibe, BD Wood. A smoothed particle hydrodynamics model for reactive transport and mineral

14 precipitation in porous and fractured porous media. Water Resour Res. 43 (2007).

15 [14] Berry RA, RC Martineau, TR Wood. Particle-based direct numerical simulation of contaminant transport and deposition in porous flow.

16 Vadose Zone Journal. 3 (2004) 164-9.

[15] Herrera PA, AJ Valocchi, RD Beckie. A multidimensional streamline-based method to simulate reactive solute transport in heterogeneous porous media. Adv Water Resour. 33 (2010) 711-27, doi: 10.1016/j.advwatres.2010.03.001.

[16] Liu MB, GR Liu. Smoothed Particle Hydrodynamics (SPH): an Overview and Recent Developments. Archives of Computational Methods in Engineering. 17 (2010) 25-76, doi: 10.1007/s11831-010-9040-7.

[17] Molins S, D Trebotich, CI Steefel, CP Shen. An investigation of the effect of pore scale flow on average geochemical reaction rates using direct numerical simulation. Water Resour Res. 48 (2012), doi: W03527

10.1029/2011wr011404.

[18] Logtenberg SA, AG Dixon. Computational fluid dynamics studies of the effects of temperature-dependent physical properties on fixed-bed heat transfer. Industrial \& Engineering Chemistry Research. 37 (1998) 739-47, doi: 10.1021/ie970382q.

[19] Morais AF, H Seybold, HJ Herrmann, JS Andrade. Non-Newtonian Fluid Flow through Three-Dimensional Disordered Porous Media. Phys Rev Lett. 103 (2009), doi: 194502

10.1103/PhysRevLett.103.194502.

[20] Dixon AG, M Nijemeisland. CFD as a design tool for fixed-bed reactors. Industrial \& Engineering Chemistry Research. 40 (2001) 5246-54, doi: 10.1021/ie001035a.

[21] Nijemeisland M, AG Dixon. CFD study of fluid flow and wall heat transfer in a fixed bed of spheres. Aiche Journal. 50 (2004) 906-21, doi: 10.1002/aic.10089.

[22] Zaretskiy Y, S Geiger, K Sorbie, M Forster. Efficient flow and transport simulations in reconstructed 3D pore geometries. Adv Water Resour. 33 (2010) 1508-16, doi: 10.1016/j.advwatres.2010.08.008.

[23] Eppinger T, K Seidler, M Kraume. DEM-CFD simulations of fixed bed reactors with small tube to particle diameter ratios. Chemical Engineering Journal. 166 (2011) 324-31, doi: 10.1016/j.cej.2010.10.053.

[24] Cardenas MB. Three-dimensional vortices in single pores and their effects on transport. Geophys Res Lett. 35 (2008), doi: L18402

$10.1029 / 2008 \mathrm{gl} 1035343$.

[25] Trebotich D, MF Adams, S Molins, CI Steefel, CP Shen. High-Resolution Simulation of Pore-Scale Reactive Transport Processes Associated with Carbon Sequestration. Comput Sci Eng. 16 (2014) 22-31.

[26] Molins S, D Trebotich, L Yang, JB Ajo-Franklin, TJ Ligocki, CP Shen, et al. Pore-Scale Controls on Calcite Dissolution Rates from Flowthrough Laboratory and Numerical Experiments. Environmental Science \& Technology. 48 (2014) 7453-60, doi: Doi 10.1021/Es5013438. 
Advances in Water Resources

[27] Celia MA, HK Dahle, SM Hassanizadeh. Dynamic pore-scale network models for two-phase flow in porous media. Computational Methods in Water Resources, Vols 1 and 2. (2000) 217-23.

[28] Gielen T, SN Hassanizadeh, MA Celia, HK Dahle, A Leijnse. A pore-scale network approach to investigate dynamic effects in multiphase flow. Computational Methods in Water Resources, Vols 1 and 2. 55 (2004) 83-94.

[29] Celia MA, PC Reeves, LA Ferrand. RECENT ADVANCES IN PORE SCALE MODELS FOR MULTIPHASE FLOW IN POROUSMEDIA. Reviews of Geophysics. 33 (1995) 1049-57, doi: 10.1029/95rg00248.

[30] Li L, CA Peters, MA Celia. Upscaling geochemical reaction rates using pore-scale network modeling. Adv Water Resour. 29 (2006) 1351-70, doi: 10.1016/j.advwatres.2005.10.011.

[31] Blunt MJ, MD Jackson, M Piri, PH Valvatne. Detailed physics, predictive capabilities and macroscopic consequences for pore-network models of multiphase flow. Adv Water Resour. 25 (2002) 1069-89, doi: 10.1016/s0309-1708(02)00049-0.

[32] Blunt MJ. Flow in porous media - pore-network models and multiphase flow. Current Opinion in Colloid \& Interface Science. 6 (2001) 197 207, doi: 10.1016/s1359-0294(01)00084-x.

[33] Blunt M, P King. Macroscopic Parameters from Simulations of Pore Scale Flow. Physical Review A. 42 (1990) 4780-7.

[34] Bijeljic B, MJ Blunt. Pore-scale modeling and continuous time random walk analysis of dispersion in porous media. Water Resour Res. 42 (2006), doi: Artn W01202

Doi 10.1029/2005wr004578.

[35] Valvatne PH, M Piri, X Lopez, MJ Blunt. Predictive pore-scale modeling of single and multiphase flow. Transport Porous Med. 58 (2005) 23 41, doi: DOI 10.1007/s11242-004-5468-2.

[36] Rhodes ME, B Bijeljic, MJ Blunt. A Rigorous Pore-to-Field-Scale Simulation Method for Single-Phase Flow Based on Continuous-Time Random Walks. Spe Journal. 14 (2009) 88-94.

[37] Al-Raoush RI, CS Willson. A pore-scale investigation of a multiphase porous media system. J Contam Hydrol. 77 (2005) 67-89, doi: 10.1016/j.jconhyd.2004.12.001.

[38] Gunjal PR, VV Ranade, RV Chaudhari. Computational study of a single-phase flow in packed beds of spheres. Aiche Journal. 51 (2005) 36578, doi: 10.1002/aic.10314.

[39] Wildenschild D, AP Sheppard. X-ray imaging and analysis techniques for quantifying pore-scale structure and processes in subsurface porous medium systems. Advances in Water Resources. 51 (2013) 217-46, doi: DOI 10.1016/j.advwatres.2012.07.018.

[40] Yang XF, TD Scheibe, MC Richmond, WA Perkins, SJ Vogt, SL Codd, et al. Direct numerical simulation of pore-scale flow in a bead pack: Comparison with magnetic resonance imaging observations. Adv Water Resour. 54 (2013) 228-41, doi: DOI 10.1016/j.advwatres.2013.01.009. [41] Scheibe TD, WA Perkins, MC Richmond, MI McKinley, PDJ Romero-Gomez, M Oostrom, et al. Pore-Scale and Multiscale Numerical Simulation of Flow and Transport in a Laboratory-Scale Column. Water Resour Res. Accepted Article (2015), doi: 10.1002/2014WR015959. [42] Molins S, D Trebotich, L Yang, JB Ajo-Franklin, TJ Ligocki, C Shen, et al. Pore-Scale Controls on Calcite Dissolution Rates from Flowthrough Laboratory and Numerical Experiments. Environ Sci Technol. 48 (2014) 7453-60, doi: 10.1021/es5013438.

[43] Trebotich D, MF Adams, S Molins, CI Steefel, S Chaopeng. High-Resolution Simulation of Pore-Scale Reactive Transport Processes Associated with Carbon Sequestration. Computing in Science \& Engineering. 16 (2014) 22-31, doi: 10.1109/MCSE.2014.77.

[44] Cirpka OA, RL Schwede, J Luo, M Dentz. Concentration statistics for mixing-controlled reactive transport in random heterogeneous media. J Contam Hydrol. 98 (2008) 61-74, doi: DOI 10.1016/j.jconhyd.2008.03.005.

[45] Luo J, M Dentz, J Carrera, P Kitanidis. Effective reaction parameters for mixing controlled reactions in heterogeneous media. Water Resour Res. 44 (2008), doi: Artn W02416

Doi 10.1029/2006wr005658.

[46] Dentz M, T Le Borgne, A Englert, B Bijeljic. Mixing, spreading and reaction in heterogeneous media: A brief review. J Contam Hydrol. 12021 (2011) 1-17, doi: DOI 10.1016/j.jconhyd.2010.05.002.

[47] Cirpka OA, EO Frind, R Helmig. Numerical simulation of biodegradation controlled by transverse mixing. J Contam Hydrol. 40 (1999) 15982, doi: 10.1016/s0169-7722(99)00044-3. 
Advances in Water Resources

[48] Cirpka OA, AJ Valocchi. Two-dimensional concentration distribution for mixing-controlled bioreactive transport in steady state. Adv Water Resour. 30 (2007) 1668-79, doi: 10.1016/j.advwatres.2006.05.022. [49] Zhang CY, K Dehoff, N Hess, M Oostrom, TW Wietsma, AJ Valocchi, et al. Pore-Scale Study of Transverse Mixing Induced CaCO3 Precipitation and Permeability Reduction in a Model Subsurface Sedimentary System. Environ Sci Technol. 44 (2010) 7833-8, doi: 10.1021/es1019788.

[50] Katz GE, B Berkowitz, A Guadagnini, MW Saaltink. Experimental and modeling investigation of multicomponent reactive transport in porous media. J Contam Hydrol. 120-21 (2011) 27-44, doi: DOI 10.1016/j.jconhyd.2009.11.002.

[51] Benson DA, MM Meerschaert. Simulation of chemical reaction via particle tracking: Diffusion-limited versus thermodynamic rate-limited regimes. Water Resour Res. 44 (2008) W12201, doi: 10.1029/2008WR007111.

[52] de Anna P, M Dentz, A Tartakovsky, T Le Borgne. The filamentary structure of mixing fronts and its control on reaction kinetics in porousmedia flows. Geophys Res Lett. 41 (2014) 4586-93, doi: Doi 10.1002/2014g1060068.

[53] de Anna P, J Jimenez-Martinez, H Tabuteau, R Turuban, T Le Borgne, M Derrien, et al. Mixing and Reaction Kinetics in Porous Media: An Experimental Pore Scale Quantification. Environ Sci Technol. 48 (2014) 508-16, doi: Doi 10.1021/Es403105b.

[54] Ding D, DA Benson, A Paster, D Bolster. Modeling bimolecular reactions and transport in porous media via particle tracking. Adv Water Resour. 53 (2013) 56-65, doi: DOI 10.1016/j.advwatres.2012.11.001.

[55] Le Borgne T, M Dentz, E Villermaux. Stretching, Coalescence, and Mixing in Porous Media. Phys Rev Lett. 110 (2013), doi: Artn 204501 Doi 10.1103/Physrevlett.110.204501.

[56] Paster A, D Bolster, DA Benson. Particle tracking and the diffusion-reaction equation. Water Resour Res. 49 (2013) 1-6, doi: Doi 10.1029/2012wr012444.

[57] Paster A, D Bolster, DA Benson. Connecting the dots: Semi-analytical and random walk numerical solutions of the diffusion-reaction equation with stochastic initial conditions. J Comput Phys. 263 (2014) 91-112, doi: http://dx.doi.org/10.1016/j.jcp.2014.01.020.

[58] Zhang CY, QJ Kang, X Wang, JL Zilles, RH Muller, CJ Werth. Effects of Pore-Scale Heterogeneity and Transverse Mixing on Bacterial Growth in Porous Media. Environ Sci Technol. 44 (2010) 3085-92, doi: 10.1021/es903396h.

[59] Acharya RC, AJ Valocchi, CJ Werth, TW Willingham. Pore-scale simulation of dispersion and reaction along a transverse mixing zone in two-dimensional porous media. Water Resour Res. 43 (2007), doi: Artn W10435

Doi 10.1029/2007wr005969.

[60] Chen L, YL He, QJ Kang, WQ Tao. Coupled numerical approach combining finite volume and lattice Boltzmann methods for multi-scale multi-physicochemical processes. J Comput Phys. 255 (2013) 83-105, doi: DOI 10.1016/j.jcp.2013.07.034.

[61] Chen L, Q Kang, B Carey, W-Q Tao. Pore-scale study of diffusion-reaction processes involving dissolution and precipitation using the lattice Boltzmann method. International Journal of Heat and Mass Transfer. 75 (2014) 483-96, doi:

http://dx.doi.org/10.1016/j.ijheatmasstransfer.2014.03.074.

[62] Cirpka OA, M Rolle, G Chiogna, FPJ de Barros, W Nowak. Stochastic evaluation of mixing-controlled steady-state plume lengths in twodimensional heterogeneous domains. J Contam Hydrol. 138 (2012) 22-39, doi: DOI 10.1016/j.jconhyd.2012.05.007.

[63] Davison SM, H Yoon, MJ Martinez. Pore scale analysis of the impact of mixing-induced reaction dependent viscosity variations. Adv Water Resour. 38 (2012) 70-80, doi: DOI 10.1016/j.advwatres.2011.12.014.

[64] Oostrom M, Y Mehmani, P Romero-Gomez, Y Tang, H Liu, H Yoon, et al. Pore-scale and continuum simulations of solute transport micromodel benchmark experiments. Comput Geosci. (2014) 1-23, doi: 10.1007/s10596-014-9424-0.

[65] Willingham T, CY Zhang, CJ Werth, AJ Valocchi, M Oostrom, TW Wietsma. Using dispersivity values to quantify the effects of pore-scale flow focusing on enhanced reaction along a transverse mixing zone. Adv Water Resour. 33 (2010) 525-35, doi: 10.1016/j.advwatres.2010.02.004. [66] Willingham TW, CJ Werth, AJ Valocchi. Evaluation of the effects of porous media structure on mixing-controlled reactions using pore-scale modeling and micromodel experiments. Environ Sci Technol. 42 (2008) 3185-93, doi: Doi 10.1021/Es7022835.

[67] Battiato I, DM Tartakovsky, AM Tartakovsky, T Scheibe. On breakdown of macroscopic models of mixing-controlled heterogeneous reactions in porous media. Adv Water Resour. 32 (2009) 1664-73, doi: DOI 10.1016/j.advwatres.2009.08.008. 
Advances in Water Resources

[68] Battiato I, DM Tartakovsky. Applicability regimes for macroscopic models of reactive transport in porous media. J Contam Hydrol. 120-21 (2011) 18-26, doi: 10.1016/j.jconhyd.2010.05.005.

[69] Boso F, I Battiato. Homogenizability conditions for multicomponent reactive transport. Adv Water Resour. 62 (2013) 254-65, doi: DOI 10.1016/j.advwatres.2013.07.014.

[70] Scheibe TD, EM Murphy, X Chen, AK Rice, KC Carroll, BJ Palmer, et al. An Analysis Platform for Multiscale Hydrogeologic Modeling with Emphasis on Hybrid Multiscale Methods. Ground Water. in press (2014), doi: 10.1111/gwat.12179.

[71] Tartakovsky AM, TD Scheibe. Dimension reduction numerical closure method for advection-diffusion-reaction systems. Adv Water Resour. 34 (2011) 1616-26, doi: 10.1016/j.advwatres.2011.07.011.

[72] Palmer B, V Gurumoorthi, A Tartakovsky, T Scheibe. A component-based framework for smoothed particle hydrodynamics simulations of reactive fluid flow in porous media. International Journal of High Performance Computing Applications. 24 (2010) 228-39, doi:

10.1177/1094342009358415.

[73] White MD, M Oostrom. STOMP Subsurface Transport Over Multiple Phases, Version 4: User's Guide. Pacific Northwest National Laboratory, Richland, Washington, 2006.

[74] Zhao Y, M Hategan, B Clifford, I Foster, G von Laszewski, V Nefedova, et al. Swift: Fast, Reliable, Loosely Coupled Parallel Computation. 2007 IEEE Congress on Services2007. pp. 199 -206.

[75] Wilde M, M Hategan, JM Wozniak, B Clifford, DS Katz, I Foster. Swift: A language for distributed parallel scripting. Parallel Comput. 37 (2011) 633-52, doi: DOI 10.1016/j.parco.2011.05.005.

[76] Scheibe TD, XF Yang, K Schuchardt, K Agarwal, J Chase, B Palmer, et al. A Many-Task Parallel Approach for Multiscale Simulations of Subsurface Flow and Reactive Transport. 7th Workshop on MTAGS New Orleans, LA, USA, 2014.

[77] Ingram GD, IT Cameron, KM Hangos. Classification and analysis of integrating frameworks in multiscale modelling. Chemical Engineering Science. 59 (2004) 2171-87.

[78] Wood BD. The role of scaling laws in upscaling. Adv Water Resour. 32 (2009) 723-36, doi: 10.1016/j.advwatres.2008.08.015.

[79] Kevrekidis IG, CW Gear, JM Hyman, PG Kevrekidis, O Runborg, C Theodoropoulos. Equation-free, coarse-grained multiscale computation: Enabling microscopic simulators to perform system-level analysis. Comm Math Sci. 1 (2003) 715-62.

[80] Tartakovsky AM, A Panchenko, KF Ferris. Dimension reduction method for ODE fluid models. J Comput Phys. 230 (2011) 8554-72, doi: 10.1016/j.jcp.2011.08.004.

[81] E WN, B Engquist, ZY Huang. Heterogeneous multiscale method: A general methodology for multiscale modeling. Phys Rev B. 67 (2003), doi: 092101 10.1103/PhysRevB.67.092101.

[82] Raicu I, I Foster, Y Zhao. Many-task computing for grids and supercomputers. IEEE Workshop on Many-Task Computing on Grids and Supercomputers (MTAGS),2008.

[83] Ogasawara E, D de Oliveira, F Chirigati, CE Barbosa, R Elias, V Braganholo, et al. Exploring many task computing in scientific workflows. IEEE Workshop on Many-Task Computing on Grids and Supercomputers (MTAGS)2009.

[84] Agarwal K, JM Chase, KL Schuchardt, TD Scheibe, BJ Palmer, TO Elsethagen. Design and Implementation of "Many Parallel Task" Hybrid Subsurface Model. MTAGS '11: Proceedings of the 2011 ACM International Workshop on Many Task Computing on Grids and Supercomputers,. Association for Computing Machinery, Seattle, WA, 2011. pp. 25-32.

[85] Gramling CM, CF Harvey, LC Meigs. Reactive transport in porous media: A comparison of model prediction with laboratory visualization. Environ Sci Technol. 36 (2002) 2508-14, doi: Doi 10.1021/Es0157144.

[86] Valdes-Parada FJ, J Alvarez-Ramirez, B Goyeau, JA Ochoa-Tapia. Jump Condition for Diffusive and Convective Mass Transfer Between a Porous Medium and a Fluid Involving Adsorption and Chemical Reaction. Transport Porous Med. 78 (2009) 459-76, doi: DOI 10.1007/s11242009-9343-z.

[87] Scheibe TD, X Yang, X Chen, GE Hammond. A hybrid multiscale framework for subsurface flow and transport simulations. Procedia Computer Science. 51 (2015) 1098-107, doi: 10.1016/j.procs.2015.05.276. 
Advances in Water Resources

1

2

4 